\title{
Morphologische Untersuchungen im nordöstlichen Matmata-Vorland (nördliche Djeffara, Südtunesien) ${ }^{1}$ )
}

\author{
Von Karl-Ulrich Brosche \& Hans-Georg Molle, Berlin
}

Mit 10 Abbildungen und 1 Tabelle

$\mathrm{Zus}$ a m menf assung. Auf Grund geomorphologischer Untersuchungen zur Abfolge der Formungsstadien im Bereich der beiden Flußsysteme Oued Termamane - Oued Zigzaou und Oued En Negueb - Oued El Hallouf - Oued Oum Ez Zessar in der nördlichen Djeffara in Südtunesien lassen sich oberhalb des rezenten Flußbetts neben jüngsten, durch menschliche Eingriffe verursachten Ablagerungen eine aus Feinsedimenten aufgebaute "Jüngere Akkumulation", eine überwiegend aus Grobschottern bestehende "Hauptakkumulation" und nicht sicher zu korrelierende Reste von zumindest zwei älteren, höher gelegenen Niveaus unterscheiden. Die "Hauptakkumulation" ist im gesamten Bereich der genannten Oueds, die "Jüngere Akkumulation“" dagegen nur in ihren Mittelund Unterläufen verbreitet. Letztere fehlt im Hauptverbreitungsgebiet der Schluffe von Matmata (primär äolisch abgelagerte und später fluviatil überformte Sedimente) im Gebirge und am Gebirgsrand. Die Ablagerung der "Jüngeren Akkumulation“ ist auf Grund der 14C-Datierung von 2 Holzkohlenproben (Hv 5566 und Hv 5400) in einen Zeitraum etwa zwischen 9000 - 7000 Jahre B.P. zu stellen, die Datierung einer Kalkkruste vom Top der „Hauptakkumulation“ ergab ein ${ }_{14}$ C-Alter von $21385 \pm 235$ Jahre B.P. (Hv 5402). Auch unter Berücksichtigung der erheblichen Fehlermöglichkeiten dieser Datierung ist ein würmzeitliches oder älteres Alter der „Hauptakkumulation" anzunehmen. Die Schluffe von Matmata überlagern die "Hauptakkumulation" und kommen als Materiallieferant für die Sedimente der „Jüngeren Akkumulation“ in Frage; daher ist mit einem würmzeitlichen bis frühholozänen Alter der Schluffe zu rechnen. Hinweise auf im Vergleich zu heute feuchtere klimatische Bedingungen geben eine fossile „Kalkbraunerde“ in den Schluffen von Matmata, das Vorkommen von Kalkkrusten und ein Schottertransport zur Zeit der „Hauptakkumulation", der erheblich weiter in das Matmatavorland reichte als der Schottertransport der rezenten Flußbetten.

$\mathrm{Su} \mathrm{m} \mathrm{m}$ a r y. Geomorphological investigations into the sequence of formation phases in the area of the two river-systems Oued Termamane - Oued Zigzaou and Oued en Negueb - Oued El Hallouf - Oued Oum Es Zessar in N. Djeffara in S. Tunisia permit us to distinguish above the recent river-bed, apart from youngest, anthropogenically caused deposits, a "younger accumulation" composed of fine sediments, a "main accumulation" consisting for the most part of coarse pebbles and remainders of at least 2 older, higher-lying levels which cannot be reliably correlated. The "main accumulation" is found in the entire area of the aforementioned Oueds, the "younger accumulation" on the other hand only in their middle and lower courses. The latter is missing in the main distribution area of the Matmata silts (primarily wind-deposited, later fluviatile remodelled sediments), in the mountains and on their edges. On the strength of the $14 \mathrm{C}$-dating of 2 charcoal specimens ( $\mathrm{Hv} 5566$ and $\mathrm{Hv}$ 5400) the deposits of the "younger accumulation" may be placed in the period between $9000-7000$ B.P. The dating of a calccrust from the top of the "main accumulation" showed a 14C-age of $21385 \pm 235$ B.P. ( $\mathrm{Hv} 5402)$. Even taking into

1) Die vorgelegte Arbeit stellt einen Teil der Untersuchungsergebnisse dar, die auf zwei Reisen nach Tunesien gewonnen wurden. Für die großzügige finanzielle Unterstützung der ersten Reise im Herbst 1972 haben wir dem Senator für Wissenschaft und Kunst in Berlin und für die Finanzierung der zweiten Reise im Sommer 1974 dem Fachbereich 24 der FU Berlin herzlich zu danken. - Herrn Dr. M. GeYh, Hannover, danken wir herzlich für die Datierung mehrerer Proben, Herrn Dr. H. Schüтt, Düsseldorf, sind wir für die Bestimmung von Mollusken zu Dank verpflichtet, ebenso Herrn Dr. G. Schulz, Berlin, der uns 1974 bei ergänzenden Untersuchungen behilflich war. 
account the considerable possibilities of error in this dating, a Wurm age may be assumed for the "main accumulation". The silts of Matmata overlie the "main accumulation" and come into consideration as supplier of material for the sediments of the "younger accumulation", accordingly the age of the silts may be assumed to be Wurm to Early Holocene. Climatic conditions more humid than those today are indicated by a fossilized "Kalkbraunerde" in the Matmata silts, the occurence of calccrusts and a transport of pebbles at the time of the "main accumulation" reaching considerably further into the Matmata foreland than the pebble-transport of the recent river-beds.

\section{Problemstellung und Einleitung}

Die Untersuchungen im Gebiet des nördlichen Vorlandes des Matmata-Berglandes in Südtunesien wurden mit dem Ziel durchgeführt, die Abfolge der für diese Region typischen Reliefstadien und Forschungsphasen zu erkennen. Dabei soll insbesondere versucht werden, die verschiedenen vorgefundenen Formungsphasen auch zeitlich einzustufen, da nur so relativ gut gesicherte Korrelationen $\mathrm{zu}$ in anderen Gebieten erarbeiteten Formungsabfolgen möglich sind. Der vorgelegten Arbeit entsprechende Untersuchungen in ausgewählten Gebieten Zentral- und Nord-Tunesiens sind im Druck bzw. in Vorbereitung (H.-G. Molle \& K.-U. Brosche 1976; K.-U. Brosche \& H.-G. Molle 1976 sowie K.-U. Brosche, H.-G. Molle \& G. Schulz 1976) und haben zum Ziel, die gegenwärtig in klimatisch sehr unterschiedlichen Regionen gelegenen Untersuchungsgebiete in bezug auf ihren Formenschatz und den in ihnen abgelaufenen Formungsphasen miteinander zu vergleichen. $\mathrm{Ob}$ sich auch Korrelationsmöglichkeiten zu Formungsabfolgen erkennen zu lassen, die in Gebieten außerhalb Tunesiens erarbeitet wurden, soll erst nach Darstellung des Formungsablaufs in Zentral- und Nord-Tunesien untersucht werden.

Die Untersuchungen wurden in der nördlichen Djeffara im Bereich folgender Oueds durchgeführt:

1. vom Oberlauf des Oued Zigzaou (= Oued Termamane) bis ans Meer;

2. vom Oberlauf des Oued en Negueb und des Oued El Hallouf, die sich zum Oued Oum Ez Zessar vereinen, bis ans Meer.

Etwas nördlich dieses Untersuchungsgebietes liegt das Becken von Toujane und Beni Zeltene, das jüngst Balland (1973) bearbeitet hat (s. u.). Durch die Ausweitung des Untersuchungsgebietes auf die Einzugsgebiete mehrerer Oueds und die Verfolgung der Oueds bis ans Meer erhofften wir uns, die Beobachtungsgrundlage gegenüber BALLAND (1973) zu erweitern und vor allem die Beziehungen zwischen den morphologischen Vorgängen im Matmata-Bergland und dem entfernteren Vorland zu erhellen sowie die morphologischen Prozesse der Jetztzeit mit vorzeitlichen Vorgängen besser vergleichen zu können.

Das Matmata-Bergland ist, wie Mensching $(1963,1964)$ ausführlich beschrieben hat, eine meist $600-700 \mathrm{~m}$ ü. M. gelegene Schichtstufenlandschaft mit nach Westen flach einfallenden Schichten. Am Aufbau sind Kalkmergel des Apt, Dolomite des Alb, Gipstone und Kalke des Cenoman, Dolomite des Tuorn und Kalke des Senon beteiligt. Gesteine der Oberkreide, die durch feste Kalke und Dolomite gekennzeichnet sind, bilden die eigentliche $200-300 \mathrm{~m}$ hohe Schichtstufe, die mit ihrer Frontstufe nach E bis ENE zeigt. Der Hauptstufenbildner ist dabei der Turonkalk. Das Gebiet des Matmata-Berglandes wurde mit dem Beginn des Tertiärs landfest. Zu einer Heraushebung, Schiefstellung und z. T. Verwerfung der Schichten der Kreide kam es im Zusammenhang mit der tertiären alpiden Tektogenese dieses Gebietes. Von dieser Heraushebung wurden auch die jurassischen Gesteine erfaßt, auf denen die Kreidegesteine diskordant auflagern. Die mächtigen Jurakalke bilden z. T. sogar eigene markante Schichtstufen (MenschING 1963, 1964). Das östliche Vorland des Matmata-Berglandes fällt von ca. 200-300 m ü.M. am Gebirgsrand 
sanft nach NE bis zum Meer ab. Es stellt geologisch eine weite Mulde dar, in der mürbe Tertiärgesteine (Gipsmergel und Mergelsande des Pont) und noch näher zu behandelnde, wahrscheinlich weitgehend quartäre Ablagerungen auftreten.

Folgende Klimadaten vermitteln eine Vorstellung vom Klima dieses Gebietes:

M a t $\mathrm{m}$ a t a (400 $\mathrm{m}$ ü. M.): Mittlerer Jahresniederschlag: etwa $240 \mathrm{~mm}$, Mittlere Jahrestemperatur: $18,9^{\circ} \mathrm{C}$; Dauer der Dürrezeit: etwa 7 Monate; $G$ a bes ( $20 \mathrm{~m}$ ü. M.): Mittlerer Jahresniederschlag: $175 \mathrm{~mm}$, Mittlere Jahrestemperatur: 19,3 ${ }^{\circ} \mathrm{C}$, Zahl der Dürremonate: 12. Die Oueds im Matmata-Vorland sollen nach Balland (1973) zwei Abkommen im Jahr erleben. Nach Mensching (1963) resultiert hieraus pflanzengeographisch eine Steppe bzw. Wüstensteppe.

\section{Literaturüberblick zum Matmata-Bergland}

Mensching (1963, 1964) gibt vor allem eine morphographisch-morphologische Analyse des Schichtstufenlandes des Matmata-Berglandes unter Herausarbeitung des aktuellen morphologischen Geschehens im Rahmen der klimatisch-morphologischen Betrachtung des Schichtstufenreliefs. Dabei beschreibt und deutet er keine Aufschlüsse und macht somit keine detaillierten Angaben zur Relief- und Klimageschichte des Matmata-Berglandes und des östlichen Vorlandes.

In einem jüngst in der DESPOIS-Festschrift (Acta Geographica 1973, Maghreb et Sahara, Etude Géographique, Paris) erschienenen Beitrag zur quartären Morphogenese im Bergland von Matmata hat Balland (1973) versucht, die Reliefgenese in dem kleinen Becken zwischen Toujane und Beni Zeltene zu klären und zu datieren.

Die Reliefformen waren in diesem Becken schon völlig im Villafranchium herauspräpariert, wie BALland (1973: 48) aus der Tatsache schließt, daß eine dicke, zweigliedrige Kruste des Villafranchium eine bereits deutlich reliefierte Oberfläche überzieht (vgl. Fig. 3, 4a, 4b, 5, 6 bei Balland 1973). Allerdings muß kritisch angemerkt werden, daß BALland keine Belege für das Alter dieser zweigliedrigen Kalkkruste anführt. Vor der Villafranchium-Krustenbildung muß eine feuchtere Phase intensiver Erosion mit kräftigen Abflüssen stattgefunden haben, in der auch das später nagelfluhartig verkrustete Grobschottermaterial aus dem Bergland von Matmata angeliefert wurde. Die Verkrustung soll bereits gleichzeitig mit der Zerschneidung der vorher abgelagerten Akkumulation begonnen haben.

Reste der „Villafranchium“-Kruste, die aus einem 30-50 cm mächtigen, sehr resistenten plattigen Teil und einem nagelfluhartig verbackenen Unterteil zusammengesetzt ist, finden sich in einer $\mathrm{j}$ ü nger e n A k k u m u lat i o n, die aus Feinmaterial, Kies, Schottern und Blöcken besteht, in aufgearbeiteter Form. Diese Akkumulation, die von BALLAND nicht datiert werden konnte, wird von einer jüngeren, schwächeren Kalkverkrustung überzogen. Die spätere Erosion, deren Alter nicht bekannt ist, hat diese Akkumulation mit der zweiten schwächeren Kruste zwar zerschnitten, die ältere „Villafranchium“-Kruste aber nach BALLAND kaum ernsthaft angegriffen, was besonders für die Nebentäler gilt. Hier hat sich die Post-,Villafranchium“-Akkumulation mit der schwächeren Kalkkruste auf die „Villafranchium“-Kruste gelegt. In den größeren Tälern dagegen ging die Post-,Villafranchium“-Erosion tiefer als in den Nebentälern. Die Post- „Villafranchium“-Akkumulation aus Feinsedimenten, Schottern, Resten der „Villafranchium“-Kruste mit einer lockeren jüngeren Kruste wird von BallaND (1973: $53 \mathrm{f}$.) wegen ihrer Ähnlichkeit zum Niveau 4 von Coque (1962) ins Amirien der marokkanischen Forscher gestellt, also in eine Phase, die dem Mindel-Glazial entspricht. 
Danach fand nach BALLAND die Akkumulation des Schluffes von Matmata statt, der sehr wahrscheinlich ein äolisches Sediment ist und alle älteren Sedimente diskordant überdeckt. Für das Alter des Schluffes fand Balland keine Anhaltspunkte. Die Lößoberfläche wurde später zu einem Glacis modelliert und es bildeten sich Kalkknollen als Indiz einer Bodenentwicklung einige Dezimeter unter der Oberfläche des Schluffes; darauf erfolgte die Zerschneidung des Schluffes z. T. in badlandartiger Weise mit dem Ergebnis, daß er mitunter nur noch in Bändern und Graten zwischen den Einschnitten erhalten ist. Das Erosionsglacis im Schluff von Matmata geht an einer Stelle hangabwärts in ein Akkumulationsglacis über. Die Bildung von Kalk-Knollen im Schluff soll ebenso einer älteren pedologischen Phase zuzuordnen sein wie eine Glasur oder Polierung auf der Oberfläche der zu dem oben genannten glacis d'érosion umgestalteten Schluffe. Die schon erwähnte Zerschneidung der Schluff-Glacis hat offenbar vornehmlich das Sediment für eine tiefste, $2 \mathrm{~m}$ Höhe nicht überschreitende Schluffterrasse geliefert. Sie enthält nicht mehr die Nodules (Kalk-Knollen), die der Schluff oberhalb dieser Terrasse aufweist. Die Schluffterrasse wird - ohne Anführung von weiteren Indizien - von Balland in das Rharbien (Postpluvial) der marokkanischen Forscher gestellt und als Niveau 1 bezeichnet. Die Umformung der Schluffe zu einem glacis d'érosion kann nach Balland zusammen mit der Bildung des Niveaus 2 von Coque (1962) im Soltanien (4. Pluvial = Würm nach den Ergebnissen der marokkanischen Forscher, vgl. Mensching 1960) entstanden sein, was die Datierung der Ausbreitung und Aufwehung der Schluffe von Matmata in die Phase 3 Coques zulassen würde, die dem Tensiftien (Riß) entspricht.

Nach BALlaRD (1973) ergeben sich nach der Villafranchium-Verkrustung folgende 4 Reliefstadien:

a) die Post-Villafranchium-Akkumulation aus Schottern, Feinsedimenten und Resten der Villafranchium-Kruste sowie die Bildung einer schwächeren Kalkkruste im A m i r i e n (Niveau 4 bei Coque, $1962=$ Mindel),

b) die Ausbreitung (vorwiegend Anwehung) der Schluffe von Matmata im Ten s if t i e n (Niveau 3 bei Coque, $1962=$ Riß),

c) die Bildung eines glacis d'érosion in diesen Schluffen im S o l t a n i e n (Niveau 2 bei Coque, $1962=$ Würm),

d) die Bildung einer Schluffterrasse im Rha rbien (Niveau 1 bei Coque, $1962=$ Postpluvial).

\section{3. "Villafranchium"-Kruste}

Die eigenen Geländebeobachtungen im östlichen Vorland des Berglandes von Matmata, in der nördlichen Djeffara, bestätigen weitgehend die Beobachtungen von BALLAND (1973) zum Formenschatz, die sich nur auf das Becken von Toujane und Beni Zeltene bis einige Kilometer ins östliche Vorland beziehen, kaum aber ihre Datierungen (s. u.).

Problematisch erscheint zunächst die „doppelgliedrige Villafranchium“-Kalkkruste, die nach BALLAND (1973) ein bereits bewegtes Relief überzieht. Nach unseren eigenen Befunden im Matmata-Bergland und seinem nördlichen Vorland findet man tatsächlich am Boden der Oueds oder wenig über dem Niedrigwasserbett durch Kalk verkrustete Schotter. Die Kalkverbackung ist stellenweise so hart, daß sie sich mit dem Geologenhammer kaum zertrümmern läßt. Jedoch gibt es u. E. keine Anhaltspunkte für ein VillafranchiumAlter dieser Kruste. Dies um so weniger, als die harte Kalkkruste, die vom Typ her der „croûte poudingiforme" von BALLAND entspricht, häufig auch mitten im Schotterkörper der „Hauptakkumulation" liegt. Diese nimmt nach unseren Untersuchungen als 4 bis 6 bis $10 \mathrm{~m}$ mächtige Schotterterrasse sowohl im Gebirge als auch im gesamten Vorland eine zentrale Stellung ein (Abb. 1,2,3). Häufig zeigte sich diese gesamte „Hauptakkumulation" 
in einer Mächtigkeit von 3-4 m völlig kalkverkrustet - allerdings nur im Gebirgsvorland (Mittel- und Unterlauf der Oueds $\left.{ }^{2}\right)$ ) und in der Nähe der Küste. Oft liegen auch mehrere Schotterhorizonte mit Kalkverbackung übereinander, die von unverbackenen Schotterlagen getrennt werden. Aus diesen Verhältnissen läßt sich u. E. schließen, d a ß in mehreren Zeiträumen die Möglichkeit zu sehr intensiven $\mathrm{K}$ a lkve rk rust u n e n der Schotterkörper bestanden haben muß. Dabei ist zunächst nur ein postpontisches Alter anzusetzen, da die Verkrustungen in Schottern vorkommen, die auf den sicher datierten Pont-Sedimenten (Mergelsande, Gipsmergel etc.) oder älteren Sedimenten der Kreide aufliegen. Auf andere Arten von Kalkkrusten wird weiter unten noch eingegangen.

Fazit dieser Ausführungen ist, daß u. E. die angebliche „Villafranchium“-Kruste BALLANDs (1973) erstens bisher nicht sicher datiert und zweitens nicht als Leithorizont für das morphologische Geschehen seit dem Villafranchium benutzt werden kann.

\section{4. „Hauptakkumulation“}

$\mathrm{Da}$ es nicht sicher erwiesen ist, ob die jüngere Reliefgeschichte des Matmata-Berglandes und seines Vorlandes mit einer „Villafranchium“-Kruste begann, die sich nach BALland (1973) nach einer bedeutenden Erosionsphase und einer folgenden Akkumulation von Schottern entwickelt haben soll, läßt sich für uns die Reliefgeschichte im Matmata-Bergland wie folgt rekonstruieren:

Beherrschend in allen drei untersuchten Tälern ist eine Schotterakkumulation, die im Gebirge bis 6-9 m mächtig werden kann, z. T. gut, z. T. schlecht geschichtet ist und im Gebirge aus sehr groben Schottern, z. T. Blöcken, aufgebaut ist (Abb. 1, 2, 3, 43)). Dieser Schotterakkumulation, die wir „Hauptakkumulation“ nennen, muß eine kräftige Eintie-

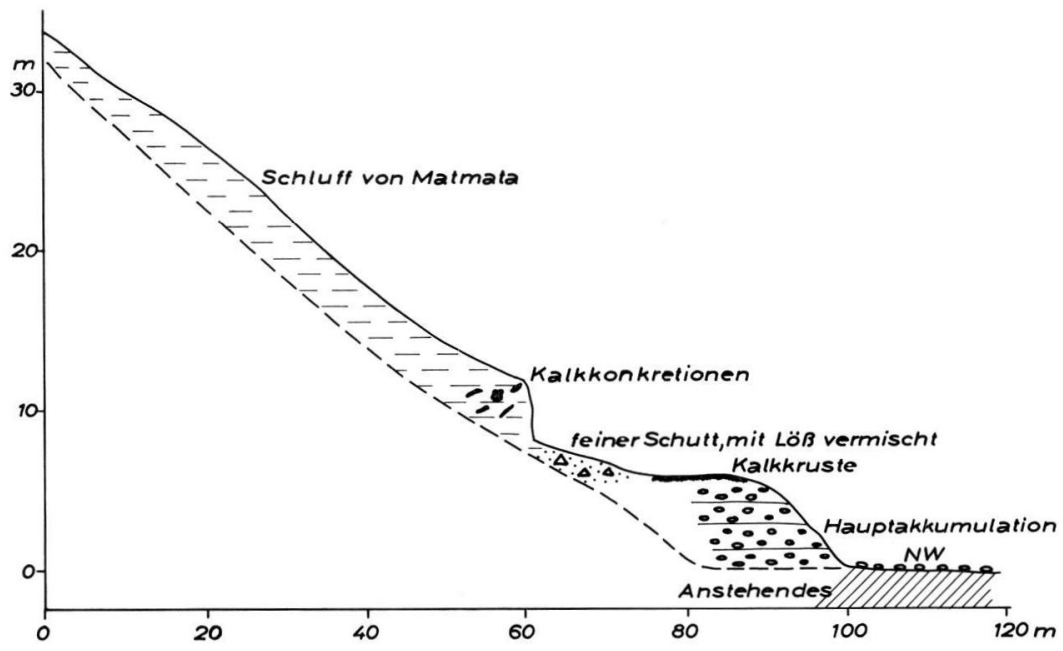

Abb. 1. W-Hang des Oued Termamane (Oberlauf des Oued Zigzaou).

2) Als Oberlauf wird der Abschnitt der Täler im Matmata-Bergland, als Mittellauf der Abschnitt vom Gebirgsrand bis zur Straße Mareth - Medenine und als Unterlauf die Strecke zwischen dieser Straße und der Küste bezeichnet.

3) In einem frisch ausgeschachteten Brunnen im oberen Mittellauf des Oued Termamane (westlich der Lokalität Roniguibat) reichte die "Hauptakkumulation“ noch ca. $10 \mathrm{~m}$ unter das Niedrigwasserbett des Oued hinab. NW bedeutet in den Abbildungen Niedrigwasserbett, HW Hochwasserbett. 
fungsphase vorausgegangen sein. Die „Hauptakkumulation“ stellt sich im Gebirge als eine durchgehend $\mathrm{zu}$ verfolgende morphologische Terrasse dar, die im Gebirge und in Gebirgsnähe von den Schluffen von Matmata (limons de Matmata) überlagert wird. Wir konnten mehrfach beobachten, daß vom Talhang ausgehende Schuttschleppen der Oberfläche der "Hauptakkumulation“ aufliegen, bzw. im oberen Horizont mit ihr verzahnt sind (Abb. 1).

Die von uns als "Hauptakkumulation" bezeichnete Akkumulation läßt sich nicht eindeutig mit einer der BaLLANDschen Akkumulationen korrelieren. Sie könnte aufgrund der schwachen Kalkverkrustung im Top der Ablagerung, die im Gebirgsabschnitt des Oued Termamane mehrfach beobachtet wurde, der jüngeren Akkumulation Ballands (1973) entsprechen (Niveau 4). Diese besteht in dem von BaLLaND untersuchten Gebiet aus Feinmaterial, Kies, Schottern, Blöcken und Resten der aufgearbeiteten „Villafranchium“-Kruste. Ferner spricht für diese Parallelisierung die Úberlagerung dieser Akkumulation durch ein Schluff-Schutt-Paket, das nach oben in reinen Schluff übergeht (vgl. Abb. 1 dieser Arbeit und Fig. 3 bei Balland). Allerdings überwiegt im Gebirgsanteil der von uns untersuchten Täler der Grobschotter- und Blockanteil deutlich gegenüber dem Feinmaterialanteil - im Gegensatz zu den Beobachtungen von BaLland (1973, Fig. 3). Jedoch stößt die Verfolgung der "Hauptakkumulation" vom Gebirge ins Vorland im Bereich der in das Gebirge eingreifenden Buchten in einem schmalen Gürtel am Gebirgsrand dadurch auf Schwierigkeiten, daß durch die hier besonders wirksame Reliefenergie auch kleine eigenständige Täler zu einer starken Überformung dieses Bereiches beitragen konnten. So wurden hier die Sedimente der "Hauptakkumulation" stellenweise ausgeräumt und die Erosionsrinnen im Zuge der folgenden Schutt- und Schluffbildungsphase (s. u. unter 5. Schluffe von Matmata) wieder verfüllt. Erst ca. $2 \mathrm{~km}$ vom Gebirgsrand entfernt läßt sich in den das Matmata-Bergland randlich gliedernden Buchten die „Hauptakkumulation“ wieder eindeutig an ihrem typischen Aufbau erkennen.

Die „Hauptakkumulation“ verfolgten wir - im Unterschied zu Balland (1973), deren Ausführungen sich nur auf den gebirgsnahen Teil beziehen, - bis an die Küste. In Richtung auf die Küste nimmt der Anteil an großen Blöcken ab. Schon 5-10 km nördlich bzw. nordöstlich des Gebirgsrandes fanden wir keine oder nur sehr wenige große Blöcke in der "Hauptakkumulation“. Während die „Hauptakkumulation“ im MatmataBergland im Sedimentkörper keine Kalkverkrustungen aufwies und lediglich an der Basis (Abb. 2) und am Top (Abb. 1) Kalkverkrustungen zeigte, stellt sich mit Entfernung vom Gebirgsrand mehr und mehr die Tendenz einer zusätzlichen Verkrustung von Teilen des Schotterkörpers oder einer Verkrustung der gesamten Grobschotterakkumulation ein. Da-

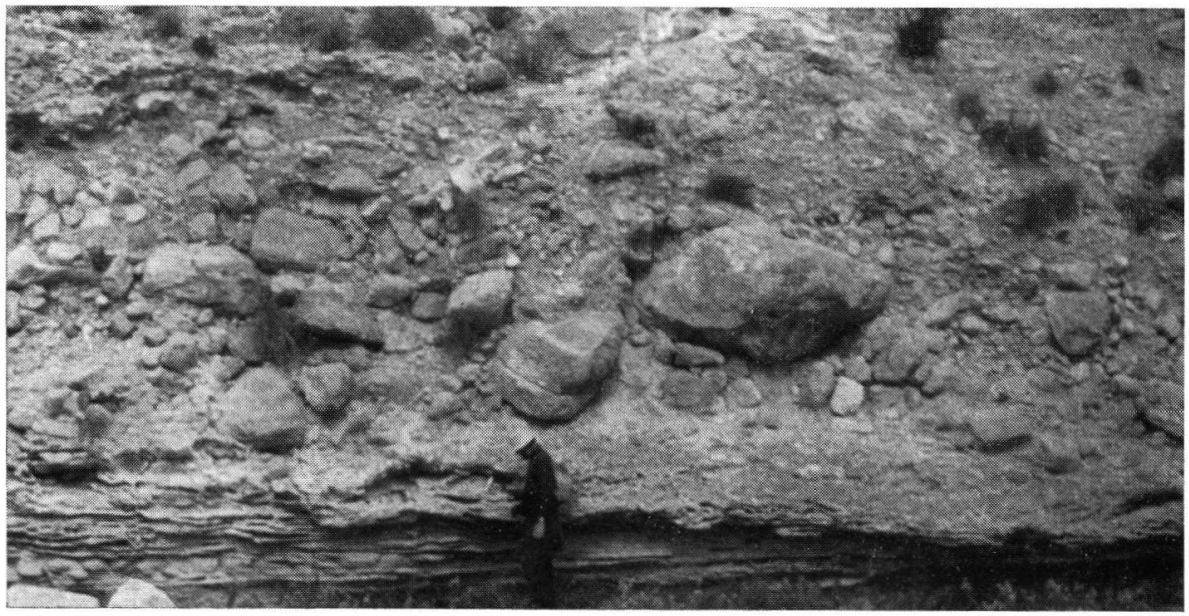

Abb. 2. „Hauptakkumulation" im Oued Termamane (= Oberlauf des Oued Zigzaou) aus groben, kantengerundeten Blöcken, Schottern, Kiesen und Sanden. Zementierte Schotter an der Basis der "Hauptakkumulation“ über anstehendem Kalk. Höhe u. M. ca. 300 m. 


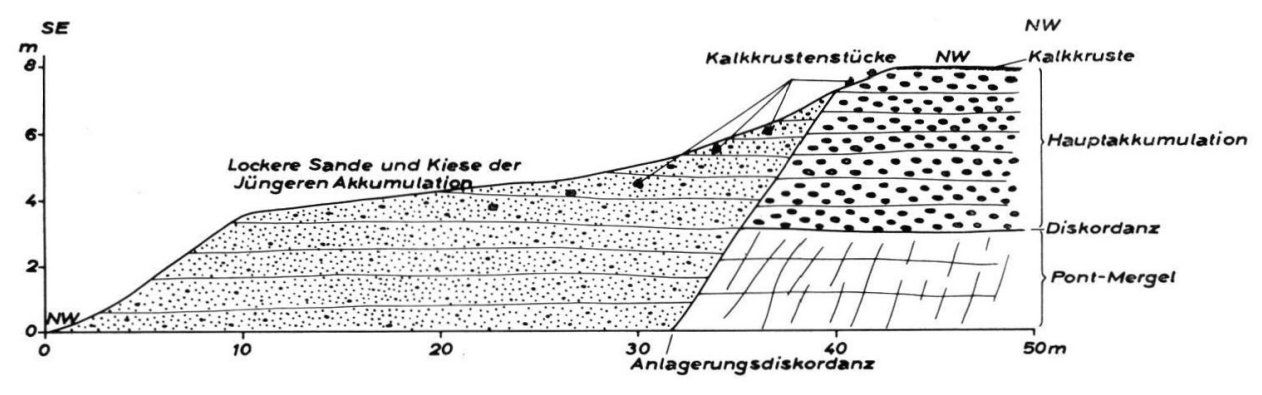

Abb. 3. Oued Zigzaou, ca. $1 \mathrm{~km}$ südlich der Straße Mareth-Medenine.

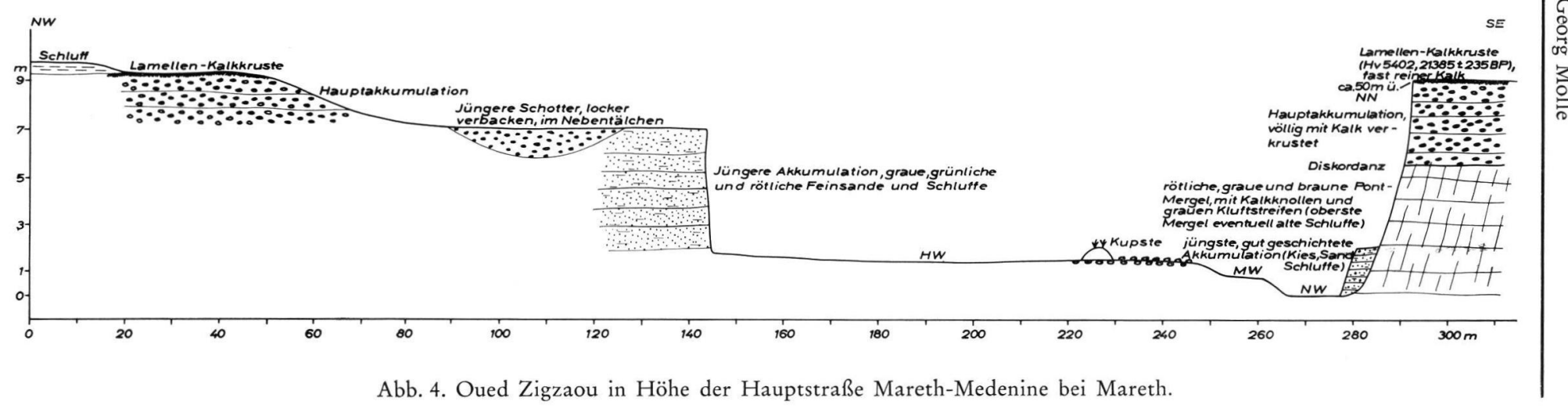


neben finden sich am Top der "Hauptakkumulation“, die das rezente Oued-Bett um $4-10 \mathrm{~m}$ überragt, häufiger fast reine Kalkkrusten, die z. T. Lamellenstruktur aufweisen, über den nagelfluhartig verkrusteten oberen Schottern der „Hauptakkumulation“.

Einen ersten Anhaltspunkt für die zeitliche Einstufung der „Hauptakkumulation “ und seiner Kalkkruste im Top erhielten wir durch eine ${ }^{14} \mathrm{C}$-Datierung einer Kruste aus überwiegend sekundärem Kalk im Top der „Hauptakkumulation“, die wir ca. $50 \mathrm{~m}$ südlich der Straße Mareth-Medenine ca. $10 \mathrm{~m}$ über dem heutigen Flußbett entnahmen. Es ergab sich bei der Probe Hv 5402 ein radiometrisches Alter von $21385 \pm 235$ B.P.

Die „Hauptakkumulation“ läßt sich im Oued El Hallouf und im Oued En Negueb, die sich zum Oued Oum Ez Zessar vereinen, sowie im Oued Zigzaou vom Gebirge bis ans Meer verfolgen. Der Schotterkörper ist meistens gut aufgeschlossen; zuweilen finden sich allerdings nur noch Schotterreste, die an das präquartäre Gestein angebacken sind. Ca. 1-1,5 km südlich des Meeres endet diese "Hauptakkumulation“ mit einem 7-8 m ü. M. gelegenen, ca. $15^{\circ}$ geneigten Kliff. Oberhalb der „Hauptakkumulation“ befindet sich in Küstennähe ein zweites Niveau in $15-16 \mathrm{~m}$ ü. M., das wie das 7-8-m-Niveau aus einer kalkverbackenen Schotterlage über anstehendem Pont-Gestein aufgebaut wird $(\text { Abb. 5 })^{4}$ ). Es ließ sich an keiner Stelle nachweisen, ob das 7-8-m-Niveau und das 1516-m-Niveau aus einem oder aus zwei verschiedenen und damit verschieden alten Akkumulationskörpern bestehen.

Noch höher als das 15-16-m-Niveau, das ebenso wie das 7-8-m-Niveau im Grundriß buchtenförmig vor- und zurückspringt, ist in Küstennähe ein $40-50 \mathrm{~m}$ hohes Niveau in Pont-Mergeln, -Gipsmergeln etc., das keine Schotter mehr trägt und durch netzförmige Gipsausblühungen und lamellenförmige Gipskrusten gekennzeichnet ist.

Während dieses 40-50-m-Niveau keine fossilen organogenen Bestandteile aufweist, fanden sich auf dem 7-8-m-Niveau ebenso reichlich Exemplare von Murex trunculus (einer marinen, im ganzen Mittelmeergebiet verbreiteten Schnecke) wie auf dem 15-16m-Niveau (Abb. 5). Die Individuen von Murex trunculus ließen sich an einer Stelle (im Unterlauf des Oued Oum Ez Zessar) kontinuierlich vom 7-8-m-Niveau bis auf das 15-16-m-Niveau hinaufverfolgen. Eine ${ }^{14} \mathrm{C}$-Datierung, die an der Probe $\mathrm{Hv} 5403 \mathrm{im}$ Unterlauf des Oued Zigzaou, ca. $500 \mathrm{~m}$ südlich der Küste auf dem 7-8-m-Niveau vorgenommen wurde, ergab ein radiometrisches Alter von $4310 \pm 170$ B.P. Ein zweites ${ }^{14} \mathrm{C}-$ Datum (Hv 6854), das ebenfalls an Schneckengehäusen von Murex trunculus, aber auf dem 15-16-m-Niveau gewonnen wurde, ergab ein ${ }^{14} \mathrm{C}$-Alter von $5725 \pm 105$ B.P.

Aus diesem Befund ist zu schließen, daß das Meer im Holozän weite Bereiche des küstennahen Teils der Djeffara überspült hat und eine sehr junge, ein bedeutendes Ausmaß annehmende holozäne Hebung des küstennahen Anteils der nördlichen Djeffara-Ebene im Raum südöstlich Mareth anzunehmen ist. Die von Autoren wie Birot (1969), Kelletat (1975), Sabelberg (1974) 5) nachgewiesenen holozänen Meeresspiegelschwankungen können solche Beträge, wie sie aus dem Vorkommen von Murex trunculus auf 15-16 m hohen Meeresterrassen zu erschließen wären, nicht erreicht haben. Nicht auszuschließen ist allerdings die Möglichkeit, daß die Salzwasserschnecken von Seevögeln auf die Abrasionsniveaus transportiert worden sind. An der heutigen Strandlinie ließen sich jedoch keine Salzwasserschnecken finden.

Auch im Bereich der Mittelläufe der Oueds ließ sich oberhalb der „Hauptakkumulation" mindestens noch ein höher gelegenes Niveau mit durch Kalk verkrusteten Schottern im Top feststellen, das im Unterschied zur "Hauptakkumulation" nicht durchgehend verfolgt werden konnte.

4) Beide Niveaus steigen vom Meer aus in Richtung S langsam an: Das untere Niveau der "Hauptakkumulation" liegt auf der Höhe von Mareth am Oued Zigzaou bei $45-50 \mathrm{~m}$ ü. M., das obere 7-8 m höher. Auf ca. $12 \mathrm{~km}$ Entfernung weisen die Niveaus somit ein Gefälle von ca. $0,3 \%$ auf.

5) Vortrag auf der DEUQUA-Tagung vom 20. - 24. 9. 1974 in Hofheim a. Taunus (Eiszeitalter u. Gegenwart 25: 210, Öhringen 1974).

15 Eiszeitalter u. Gegenwart 


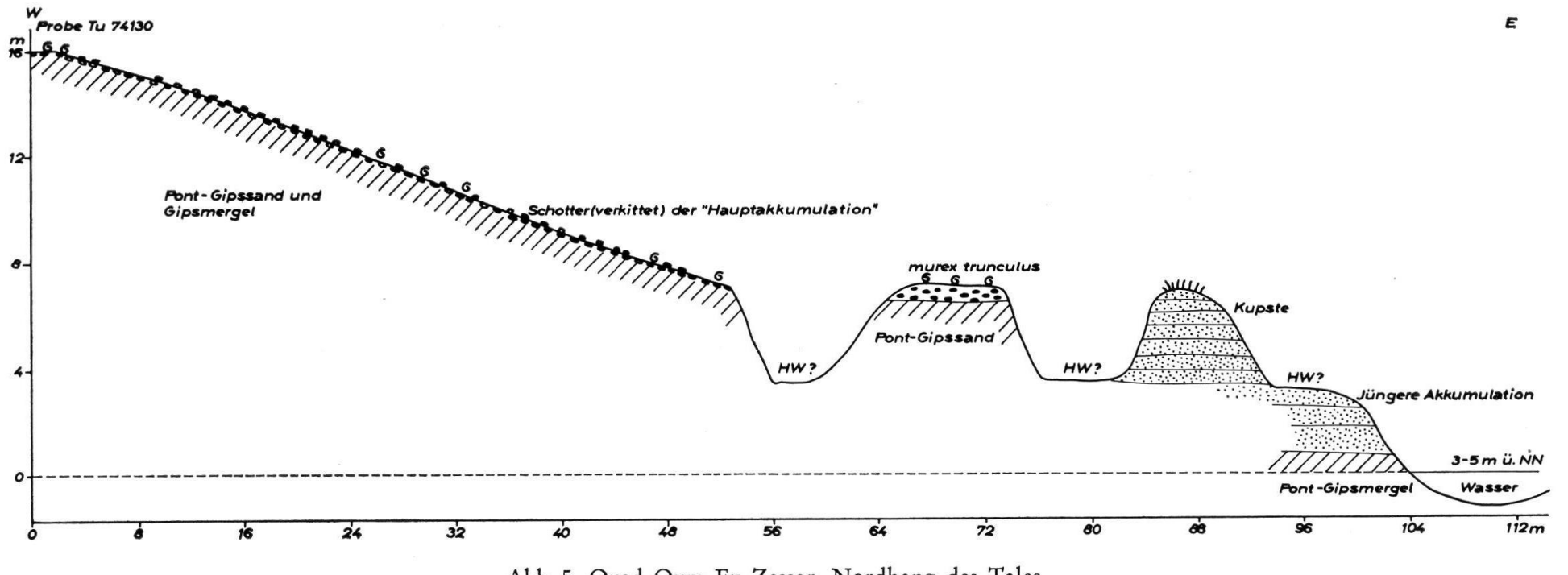

Abb. 5. Oued Oum Ez Zessar, Nordhang des Tales. 


\section{Die Schluffe von Matmata und ihre Überformung}

Eindeutig jünger als die „Hauptakkumulation“ sind die Schluffe von Matmata (limons de Matmata), die ähnlich wie Löß Wände bilden und bereits von RATHJENs sen. (1928), Schwegler (1944), Le Houérou (1960), Brunnacker (1973) und Balland (1973) erwähnt bzw. beschrieben werden. Sie legen sich im Gebirgsabschnitt der drei bis in das Gebirge verfolgten Täler deutlich auf die heute in 5-10 m Mächtigkeit aufgeschlossene „Hauptakkumulation" auf (Abb. 1). Nach Ablagerung der Schluffe wurden diese und die darunter liegende „Hauptakkumulation“ zerschnitten. Ob bereits eine Zerschneidung der "Hauptakkumulation“ vor Ablagerung der Schluffe stattfand, ließ sich bisher nicht feststellen. Ihre größte Mächtigkeit erreichen die Schluffe von Matmata im Gebirgsteil der Täler, am Gebirgsrand und an den Flanken niedriger Hügel und Ketten in Gebirgsnähe. In langen Hangschleppen ziehen die Schluffe hier mehrere Dekameter, z. T. über $50-70 \mathrm{~m}$ hoch die Steilhänge der Täler empor und lassen dadurch bereits ihre äolische Entstehung erkennen (vgl. auch Balland 1973). Es zeigte sich, daß die Schluffe auf den Südhängen der Oueds, die im Lee zu den südwestlichen, aus dem Grand Erg Oriental kommenden Winden liegen, höher hinaufreichen als auf den Nordhängen. Dabei dünnen die Schluffe, wie in Abb. 1 dargestellt, hangaufwärts mit dem Steilerwerden der Hänge aus. Die Anlieferung der Schluffe erfolgte durch äolischen Transport. Dies schließt allerdings nicht aus, daß stellenweise eine Verschwemmung oder eine andere nichtäolische Verlagerung der Schluffe stattgefunden hat (Deluviallöß bei BrunNACKer 1973). (Die äolische Anwehung von Schluffen und Feinsanden aus dem Grand Erg Oriental von Südwesten her geht auch heute noch vor sich. Eine äolische Akkumulation (Flugsandfelder, Dünenentstehung) und Deflation (Bearbeitung von Kupsten) ist im gesamten Untersuchungsgebiet bis an die Küste zu beobachten).

Im Gegensatz zum gebirgsnahen Teil des Matmata-Berglandes und zum MatmataBergland selbst finden sich auf den Flächen zwischen den untersuchten Oueds im Bereich ihrer Mittel- und Unterläufe meist nur $0,8-1,2 \mathrm{~m}$, stellenweise auch $2-3 \mathrm{~m}$ mächtige Schluffdecken mit der typischen gelblichen Lößfarbe. Allerdings gelang es bisher noch nicht, die dünnen Schluffdecken des Gebirgsvorlandes zeitlich mit den mächtigen Schluffdecken ihres Hauptverbreitungsgebietes im Gebirge und am Gebirgsrand zu parallelisieren.

Zwei ${ }^{14} \mathrm{C}$-Datierungen an Mollusken im Vorland geben jedoch wenigstens für diese Schluffe Anhaltspunkte für ihr Alter: Die erste Probe Hv 5401 stammt aus Feinsanden und Schluffen im Top der unten noch näher zu behandelnden "Jüngeren Akkumulation“ im Unterlauf des Oued En Negueb kurz oberhalb der Vereinigung des Oueds mit dem Oued El Hallouf (Abb. 6). Sie ergab ein radiometrisches Alter von $8975 \pm 215$ B.P. Dieses Alter ist allerdings, wie sich im Vergleich mit zwei Holzkohledaten unter dieser Schneckenprobe zeigt (s. u.), eindeutig zu hoch. Vielmehr müßte ein Alter von weniger als $7775 \pm 340$ B.P. richtig sein. Die Erklärung für das zu hohe Alter der Molluskenprobe dürfte in erster Linie darin zu suchen sein, daß die Mollusken Kalk aus älteren Formationen aufgenommen und mit zum Aufbau ihrer Gehäuse verwandt haben. Eine zweite Schneckenprobe (Hv 5404), die ebenfalls im Deckschluff und zwar hier über der „Hauptakkumulation" ca. $5 \mathrm{~km}$ nördlich der Straße Mareth-Medenine auf einem Plateau auf der Ostseite des Oued Oum Ez Zessar entnommen wurde, ergab ein recht ähnliches radiometrisches Alter, nämlich von $8315 \pm 120$ B.P. Auch diese Datierung dürfte ein zu hohes Alter vortäuschen.

$\mathrm{Da}$ sich aber der Schluff im Gebirge und am Gebirgsrand bisher nicht datieren ließ und kein organisches Material in ihm zu finden war, muß sein genaues Alter weiterhin als unbekannt gelten. Aus dem Gesamtzusammenhang und der Interpretation des noch darzustellenden jüngeren Formenschatzes werden einige Vorstellungen zu seinem Alter zu entwickeln sein. 


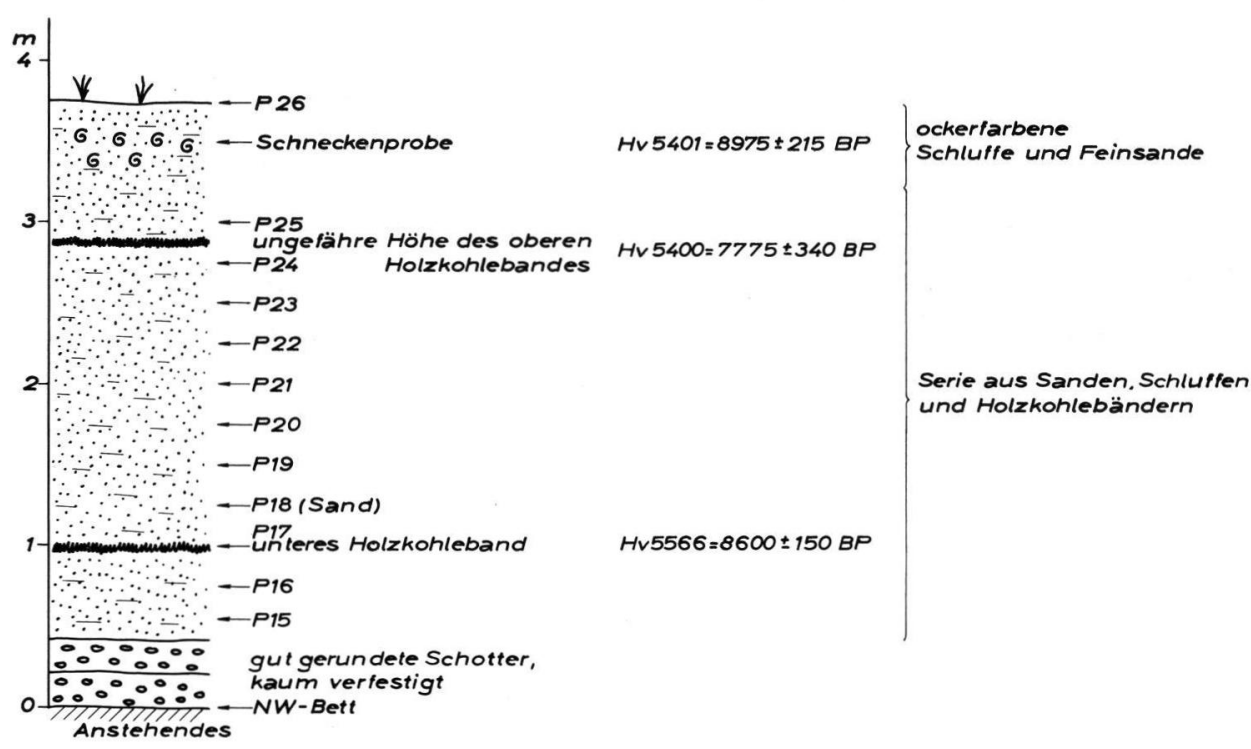

Abb. 6. Oued En Negueb (oberhalb der Vereinigung mit dem Oued El Hallouf) Aufschluß in "Jüngerer Akkumulation“.

Der Schluff von Matmata zeichnet sich, wie schon Balland (1973) erwähnt, z.T. durch eine Beimengung von Hangschutt aus. Abb. 7, die im Oberlauf des Oued Zigzaou in einer Hangmulde aufgenommen wurde, läßt zunächst wie Abb. 1 erkennen, daß der Schluff von Matmata besonders an der Basis über der "Hauptakkumulation“ durch ein Schutt-Schluff-Gemisch ausgezeichnet ist. Darüber folgt ein mehr hellgrauer Schluff mit wenigen kantigen Steinen, darüber eine von Schluff durchsetzte zweite Schuttlage und darüber wiederum ein schwach-rötlich-gelber Schluff ohne oder fast ohne Steine ${ }^{6}$ ). Dieses obere Schluffpaket ist im höheren Teil (in den oberen $1,5 \mathrm{~m}$ ) frei von Kalkkindeln bzw. -knollen. Charakteristisch für diesen oberen Schluff ist eine schwache $\mathrm{r}$ ö t li c h - b r a u n e V e rf ärbung mit Kalkkindeln im Liegenden, die als schwache Bodenbildung zu deuten ist. Diese schwache Bodenbildung hat auch bereits Brunnacker (1973: 92) nördlich Matmata an der Straße Matmata-Gabes gefunden und als Kalkbraunerde angesprochen.

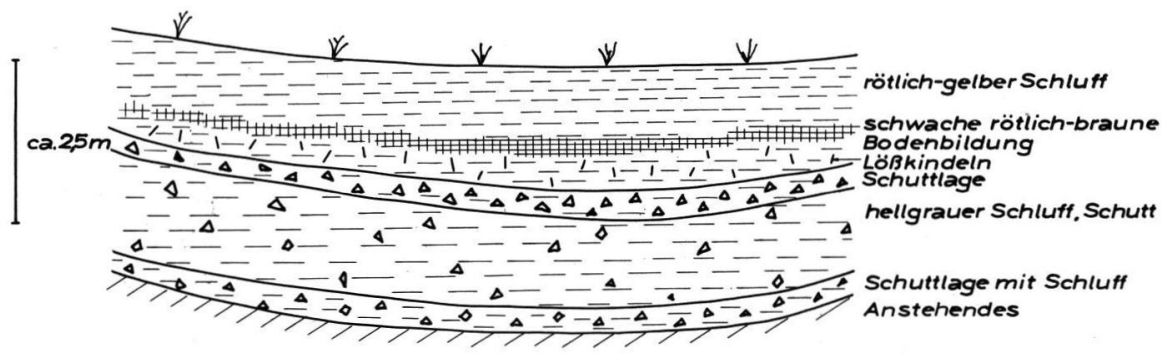

Abb. 7. S-Hang des Oued Termamane.

6) Rathjens (1928: 222) hebt gleichfalls hervor, daß der von ihm untersuchte Löß am Djebel Gharian (Libyen) eine Zweiteilung auf weist: „Fast überall, wo ich diese Höhlen sah, lagen unter dem typischen rötlich-gelben Löß festere, hellere, mit Kalkkonkretionen gefüllte Massen, die die Basis der Lößdecke zu bilden scheinen." 
BALLAND (1973) erwähnt diese schwache rötlich-braune Bodenbildung nicht, hebt aber mehrfach hervor, daß sich pedogenetische Überprägungen in Form von Schluffkindelzonen und, wenn der Schluff in seinen oberen Schichten flächenhaft abgetragen ist, in Form einer Glasur bzw. Polierung der Oberfläche darstellen.

Diese rötlich-braune, mit Brunnacker (1973) als fossile Bodenbildung gedeutete Verfärbung im Schluff von Matmata kommt im Gebirgsanteil aller von uns untersuchten Täler des Berglandes von Matmata, dazu noch im Becken nördlich von Beni Kheddache im Oberlauf des nach Westen ziehenden Oued Bel Krecheb vor.

Im Oberlauf des Oued En Negueb tritt die schwache Bodenbildung ca. $1 \mathrm{~km}$ vom Gebirgsrand entfernt im Gebirge am Südhang des Oued auf. Hier finden sich unter der rötlich-braunen Verfärbung sogar plattige dünne Kalkkrusten von 5-6 cm Dicke, die die Schluffkindeln vertreten. (Ahnliche plattige Kalkanreicherungen kommen auch häufiger am Gebirgsrand am Rande des Oued Termamane vor.)

Im Oberlauf des Oued El Hallouf ist die fossile Bodenbildung in einer Talerweiterung direkt unterhalb von Ksar El Hallouf zu beobachten, wo die Schluffe südöstlich der Oase unterhalb der verlassenen Ghorfa-Siedlung Mächtigkeiten von über 5-8 m erreichen und nach ihrer Ablagerung stark linienhaft und flächenhaft abgetragen worden sind.

Auch wenn die schwache Bodenbildung bisher nicht datierbar ist, so zeigt sie doch an, daß bei ihrer Bildung offenbar etwas feuchtere Klimabedingungen als gegenwärtig geherrscht haben müssen; denn am Top der Schluffe läßt sich heute keine Bodenbildung beobachten (so auch BRUNNACKER 1975 7)); vielmehr herrscht eine äolische und fluviale Überprägung vor. Ferner zeigt die schwache fossile pedogenetische Überprägung an, daß es im Gebirgsbereich wenigstens zwei bedeutende Schluffanwehungs $\mathrm{ph}$ a s e $n$ gegeben haben muß, wobei sich die jüngere Phase am ehesten mit den dünnen, mit Hilfe von zwei Schneckenproben datierten Deck-Schluffen des nördlichen MatmataVorlandes parallelisieren lassen dürfte.

Ob man die Abb. 7 aufgrund der Einschaltung eines deutlichen Schuttbandes in der Mitte des Schluffes noch weiter klimatisch-morphologisch ausdeuten kann, wagen wir nicht $\mathrm{zu}$ entscheiden. Auch heute läßt sich stellenweise eine Bewegung von Schuttpartikeln auf den steilen Talhängen im Anstehenden und auf den Schluffen beobachten. Neben einer

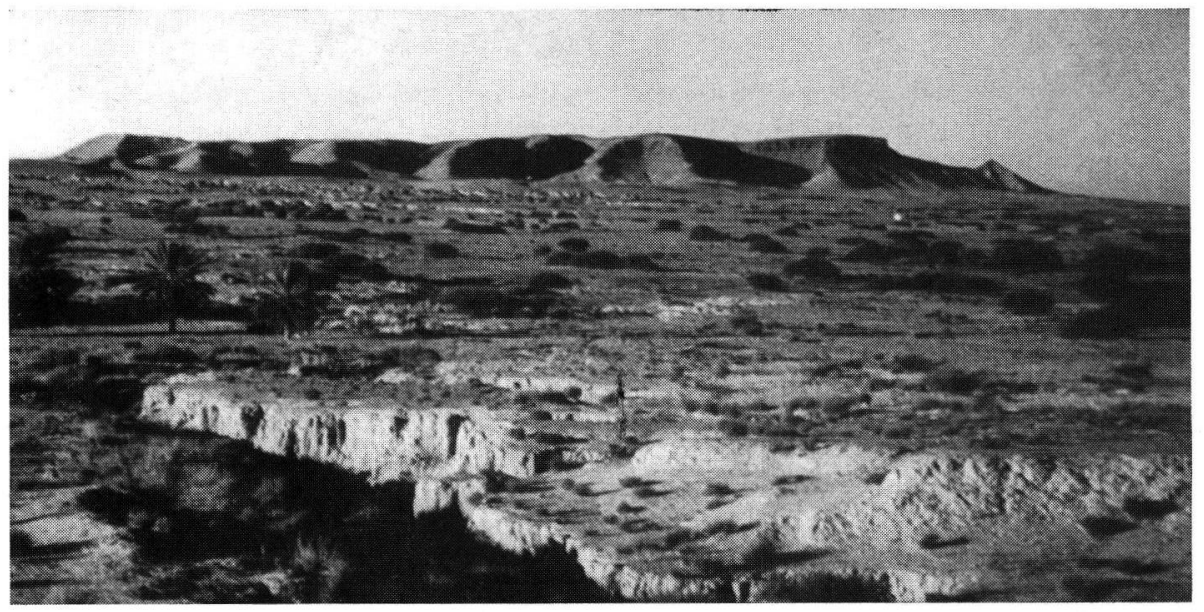

Abb. 8. Zerkerbte Schluffe von Matmata im Vordergrund und am Fuß der Schichtstufe im Hintergrund in der Bucht zwischen dem Djebel Tebaga und dem Djebel Bateun Krada (Oued Termamane/Oberlauf des Oued Zigzaou). Bildmitte ca. $180 \mathrm{~m}$ ü. M.

7) In einer Antwort auf eine Diskussionsfrage von K. U. BRosche im Berliner Geographischen Colloquium am 6. 2. 1975. 
solchen, vor allem auf fluviale Tätigkeit zurückzuführenden Schuttbewegung findet heute eine Zerkerbung der Hang-Schluffe statt (Abb. 8). Daß z. Z. eine mechanische Schuttaufbereitung erfolgt, hat bereits MensCHING (1963) hervorgehoben.

Die Schluffe von Matmata sind, wie BALland (1973) bereits ausführlich beschrieben hat, am Gebirgsrand, in Talerweiterungen und am Fuße von kleinen Vorhügeln und Rükken nahe des Gebirgsrandes häufig flächenhaft abgetragen worden, wobei glacisartige Geländeteile entstanden sind (Abb. 8). Das Ergebnis dieser Abtragung ist die Entfernung des oberen kalkkindelfreien gelblich-rötlichen Schluffes, wie z. B. häufiger am Gebirgsrand nahe dem Oued Termamane. Auch kommt es im Zuge der Überarbeitung der Schluffe vielfach zu einer Ablösung der Schluff-Glacis bzw. Schluff-Hangschleppen vom Hang. Diese auf die größeren Täler zu abfallenden glacisartigen Formen im Schluff (glacis d'érosion nach BALLAND) sind bereits wieder deutlich durch eine rezente kräftige Zerkerbung in Auflösung begriffen (Abb. 8). Balland (1973) datiert diese Glacisbildung im Schluff von Matmata, in deren Gefolge Lößkindelhorizonte und durch Pedogenese entstandene Glasuren an die Oberfläche traten, ins Soltanien (Niveau 2 bei CoQUe $1962=$ Würm), ohne aber dafür Belege anzuführen. Die Anwehung des Schluffes von Matmata dagegen datiert sie ins Tensiftien (Niveau 3 bei Coque $1962=$ Riß). Die Bildung der jüngsten Reliefform, einer Schluffterrasse von 1-2 m Höhe, die Balland (1973) mit dem Niveau 1 von CoQue (1962) vergleicht, stellt sie ins Rharbien = Postpluvial.

Die Annahme einer eigenen langen vorzeitlichen Formungsphase (Würm), die zur Ausbildung des glacis d'érosion in den Schluffen von Matmata geführt haben soll, ist u. E. nicht gerechtfertigt. Vielmehr läßt sich aufgrund der dargelegten Befunde eine linien- und z. T. flächenhafte Überformung der Schluffe in Gebirgsnähe heute noch beobachten; gegenwärtig scheint jedoch die linienhafte Zerschneidung der Schluffe zu überwiegen. Die flächenhaften Anteile des glacis d'érosion nehmen nur einen relativ kleinen Raum zwischen den Kerben ein.

Gegen die von Balland (1973) ins Würm gestellte Bildung der glacis d'érosion im Schluff von Matamata spricht, daß BALLAND (1973: 56) selbst beschreibt, wie an einer Stelle ein glacis d'érosion in ein glacis d'accumulation übergeht. Das gibt bereits einen Hinweis darauf, daß durch die Überformung der Schluffe von Matmata Akkumulationsformen entstanden sind.

\section{6. "Jüngere Akkumulation"}

Nach unseren Untersuchungen kann hauptsächlich die äolische und fluviale Überformung der Schluffe von Matmata das Material für eine jüngere, überwiegend gut geschichtete Feinmaterialakkumulation geliefert haben, die sich erst in $4-5 \mathrm{~km}$ Entfernung vom Gebirgsrand als eigenständige, streckenweise wieder aussetzende Akkumulationsterrasse zu erkennen gibt. Im unteren Mittellauf und im Unterlauf der Täler ist sie fast durchgehend gut entwickelt und überragt hier das Niedrigwasserbett meist um 3-6 m, im Unterlauf z. T. um 8-10 m (Abb. 9). Diese weitgehend aus schwach rötlich bis gelblich gefärbten Schluffen und Feinsanden aufgebaute Akkumulationsterrasse, die z. T. aber auch besonders an der Basis - aus lockeren, unverfestigten Grobsanden, Kiesen und Schottern aufgebaut ist, nennen wir die "Jüngere Akkumulation“. Es handelt sich dabei um die bereits von LE HouḱRou (1960) erwähnte Terrasse, die aber von ihm noch nicht datiert werden konnte.

Im Mittellauf der Täler konnte mehrfach beobachtet werden, daß während des Aufbaus der "Jüngeren Akkumulation“ eine Zufuhr von Material (Schluffe, Sande, Kiese) von den Hochflächen zwischen den größeren Oueds her erfolgte. Das Material wurde über Muldentäler, die in der „Hauptakkumulation“ und dem darüber liegenden Relief ent- 


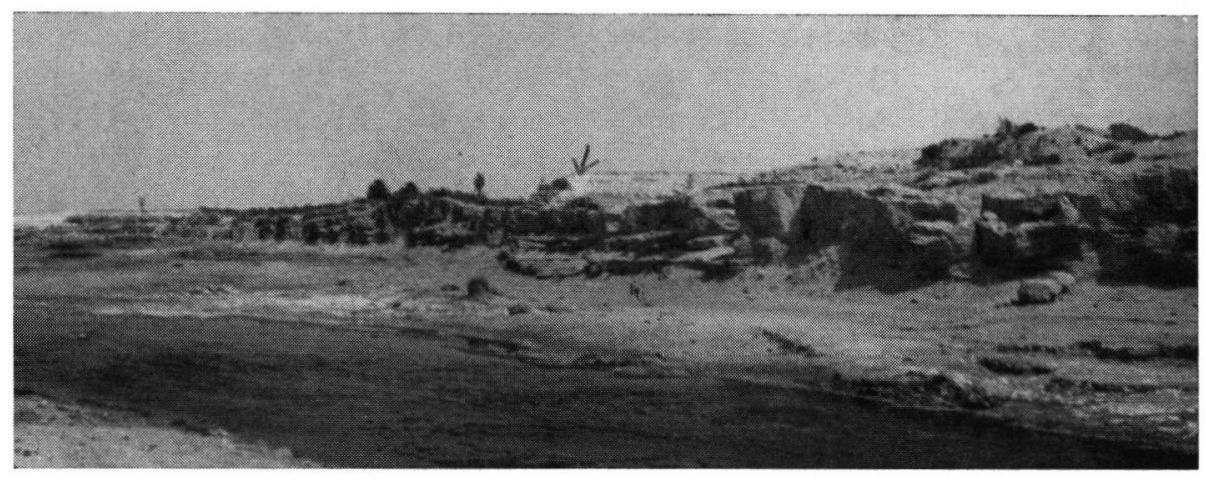

Abb. 9. Gut geschichtete Feinsedimente der „Jüngeren Akkumulation“ vor der „Hauptakkumulation“ (Pfeil) im Unterlauf des Oued Oum Ez Zessar.

wickelt sind, der "Jüngeren Akkumulation“ zugeliefert. Die beschriebenen Grobsand-, Kies- und Schotteranteile weist diese "Jüngere Akkumulation" vornehmlich im Mittellauf auf (Abb. 6). Diese "Jüngere Akkumulation" kommt in allen untersuchten Tälern vor und zeichnet sich im unteren Mittellauf und im Unterlauf durch ein bis zwei, im Aufschluß auf- und abschwingende graue Lehmbänder und durch ein fast durchlaufendes $\mathrm{Hol}$ z k o hleband aus. Außerdem ist sie stellenweise im Unterlauf durch kleine Kalkstengel oder -röhrchen charakterisiert. Im Unterlauf reicht die "Jüngere Akkumulation“ also maximal bis in die Höhe der "Hauptakkumulation“. Hier bestehen die oberen 4-6 $\mathrm{m}$ der "Jüngeren Akkumulation" fast ausschließlich aus feinen Sanden.

Die oberen 4-6m dieser Lockersedimente sind bereits bzw. werden gegenwärtig hauptsächlich äolisch abgetragen (Abb.9) und in Richtung auf die Wattenküste verfrachtet. Eine Abtragung durch Kerbtälchen, die auf das Hauptoued eingestellt sind, findet außerdem gegenwärtig statt.

An einer Stelle (am Oued En Negueb kurz oberhalb vor seiner Vereinigung mit dem Oued El Hallouf) sind $\mathrm{zwei} \mathrm{Holzk}$ oh le bänder entwickelt. Streckenweise folgt als Abschluß dieser "Jüngeren Akkumulation“ ein gelblich-rötlicher Schluff, in dem die bereits oben erwähnte Schneckenprobe entnommen und datiert wurde, die ein radiometrisches Alter von $8975 \pm 215$ B.P. ergab ( $\mathrm{Hv}$ 5401). Zwei weitere ${ }^{14} \mathrm{C}-\mathrm{Daten}$, die an der gleichen Aufschlußwand an Holzkohleproben gewonnen wurden (Hv 5566 und $\mathrm{Hv}$ 5400), lauten: $8600 \pm 150$ B.P. (die Probe wurde 1,0 m über dem Niedrigwasserbett entnommen) bzw. $7775 \pm 340$ B.P. (2,5-3,0 m über Niedrigwasserbett, Abb. 6). Damit dürfte diese "Jüngere Akkumulation" mit der „basse terrasse" von Coque (1962) vergleichbar sein, die dieser in seinem weiter nördlich gelegenen Arbeitsgebiet gefunden und in das Capsien gestellt hat.

Im unteren Mittellauf des Oued Zigzaou, ca. $1 \mathrm{~km}$ südlich der Straße Mareth-Medenine, ist die "Jüngere Akkumulation" überwiegend aus Feinsanden aufgebaut (Abb. 3 u. 4). Sie weist hier eine deutliche Anlagerungsdiskordanz ${ }^{8}$ ) an die „Hauptakkumulation“ auf (Abb. 3) und enthält mehrere Stücke einer auf der „Hauptakkumulation" ausgebildeten schwächeren Kalkkruste. Diese Kalkkruste überzieht selbst den Boden eines in die "Hauptakkumulation" eingelassenen Muldentälchens, das mit Lockermaterial der "Jüngeren Akkumulation" ausgefüllt war. Diese Beobachtung läßt sich dahingehend ausdeuten, daß es während oder nach der Erosionsphase nach Ablagerung der „Haupt-

8) Eine ganz ähnliche Anlagerungsdiskordanz entdeckten wir auch im Mittellauf des Oued El Hallouf. 
akkumulation“ zur Bildung von Lamellen-Kalkkrusten und einer Kalk-Verkittung von Schottern kam.

Die partielle Abtragung der Sedimente der "Jüngeren Akkumulation" und ihre Zerschneidung durch die Haupt-Oueds kann aufgrund der oben genannten ${ }^{14} \mathrm{C}$-Daten nach ca. 7700 B.P. eingesetzt haben.

Im untersten Unterlauf des Oued Oum Ez Zessar hat sich im Rahmen der bereits erwähnten Tieferschaltung der "Jüngeren Akkumulation“ ein "Zwischenniveau“ 3,5-4 m über Niedrigwasserbett eingestellt, auf dem sich 3-4,5 m mächtige, mit Tamarisken besetzte Kupsten bis heute erhalten haben (Abb.5). Möglicherweise zeigen die Tops der Kupsten in etwa noch die ehemalige maximale Sedimentationshöhe der „Jüngeren Akkumulation “ an. Das braucht aber nicht unbedingt der Fall zu sein, denn die Kupsten treten auch auf allen anderen Reliefteilen auf, die oberhalb des Niedrig- und Mittelwasserbettes liegen.

Die vorwiegend äolische Abtragung der „Jüngeren Akkumulation“ und äolische Prozesse nehmen in Küstennähe bzw. in Annäherung an das Garaet Bel (auch Sebkra Oum Ez Zessar genannt), in das das Oued Ez Zeuss und das Oued Oum Ez Zessar auslaufen, ein bedeutendes Ausmaß an: Die "Jüngere Akkumulation“ überragt hier das Niedrigwasserbett $\mathrm{z}$. T. nur noch um 1-2 m; außerdem kommt es hier zur Bildung von Miniaturbarchanen und unregelmäßigen Sandanhäufungen. Die „Jüngere Akkumulation “ läßt sich jedenfalls - wohl wegen der starken rezenten Windaktivität - in keinem der untersuchten Täler mehr bis ans Meer verfolgen: Sie setzt allmählich aus bzw. taucht mit ihren unteren Schichten in das Garaet Bel ab.

Ein ähnliches Zwischenniveau wie im Unterlauf des Oued Zigzaou und Oued Oum Ez Zessar weist das Oued Zigzaou auch im unteren Mittellauf südlich Mareth auf. Vor allem durch äolische Abtragung ist hier die 2-3 m mächtige, aus Schluffen und Feinsanden aufgebaute Deckschicht der "Jüngeren Akkumulation“, die in diesem Talabschnitt auf locker gelagerten Sanden, Kiesen und Schottern der gleichen Akkumulationszeit liegen, wieder abgetragen worden. Ergebnis dieser Abtragung ist nicht nur eine zusätzliche morphologische Terrasse, sondern auch ein $S$ te in pflaster über dem unteren Niveau der "Jüngeren Akkumulation", auf dem nach einer freundlichen Bestimmung des von uns aufgesammelten Materials durch Herrn B. Gabriel, Stuttgart, neolithische Artefakte auftreten. Diese und das Steinpflaster setzen heute der weiteren äolischen Überformung einen gewissen Widerstand entgegen und geben damit einen Hinweis darauf, daß hier die Überformung der "Jüngeren Akkumulation" bereits recht früh eingesetzt haben kann.

\section{7. „Jüngste Akkumulation“}

Als eine "Jüngste Akkumulation" lassen sich vorwiegend schluffige Sedimente im oberen Mittellauf der untersuchten Täler ausgliedern, die dort nahe dem Gebirgsrand in künstlich durch Steinwälle verbauten Nebentälern erscheinen. Es ist wahrscheinlich, daß auch eine jüngste 1,0-1,5 $\mathrm{m}$ hohe Terrasse in diesen Talabschnitten rezenten bzw. subrezenten Alters ist. Das in ihr abgelagerte feine Material, das sich in erster Linie aus der Abtragung der Schluffe von Matmata herleiten läßt, wird nahe dem Gebirgsrand häufig durch künstliche Mauern festgehalten und stellt die Grundlage für einen Bewässerungsfeldbau dar.

Bei der rechts in Abb. 4 sichtbaren "Jüngsten Akkumulation“ im Oued Zigzaou handelt es sich ebenfalls um eine rezente bzw. subrezente Bildung, die aus Schluffen, Sanden, Kiesen und Schottern aufgebaut ist. Sie überragt um 1,5-1,7 $\mathrm{m}$ das Niedrigwasserbett, ist völlig locker gelagert und dürfte durch Hochwässer des Oued Zigzaou heute noch erreicht werden. Etwa bis zur gleichen Höhe über das NW-Bett reichen in diesem Talab- 
schnitt nämlich Kies und grobe Schotter des Hochwasserbettes des Oued Zigzaou (Abb. 4). Nicht auszuschließen ist, daß diese "Jüngste Akkumulation" hier durch den Stau, den die Straße Mareth-Medenine auf das morphologische Geschehen im Oued Zigzaou ausübte, entstanden ist. Diese Akkumulation lagert sich stellenweise an die "Jüngere Akkumulation" und ist daher jünger als diese.

Während den bisher behandelten jüngsten Akkumulationen eher ein subrezentes Alter zukommen dürfte, läßt sich im heutigen Flußbett vom Gebirge bis zum Meer eine typische Sedimentabfolge feststellen: Im Oberlauf und im oberen Mittellauf handelt es sich in erster Linie um Grobschotter und Blöcke bis zu $>1 \mathrm{~m} \phi$ auf Anstehendem oder auf Resten der „Hauptakkumulation“. Darauf folgt im mittleren Mittellauf eine Strecke, in der Kiese und grobe Schotter dominieren. Hier kann der Höhenunterschied zwischen diesen rezenten Akkumulationen und den älteren Akkumulationen („Jüngere Akkumulation “ und „Hauptakkumulation“) streckenweise nur 1-2,5 m betragen. Es ist dies im Oued Zigzaou ein Talabschnitt, in dem heute ein äußerst intensiver Schottertransport im rezenten OuedBett stattfindet.

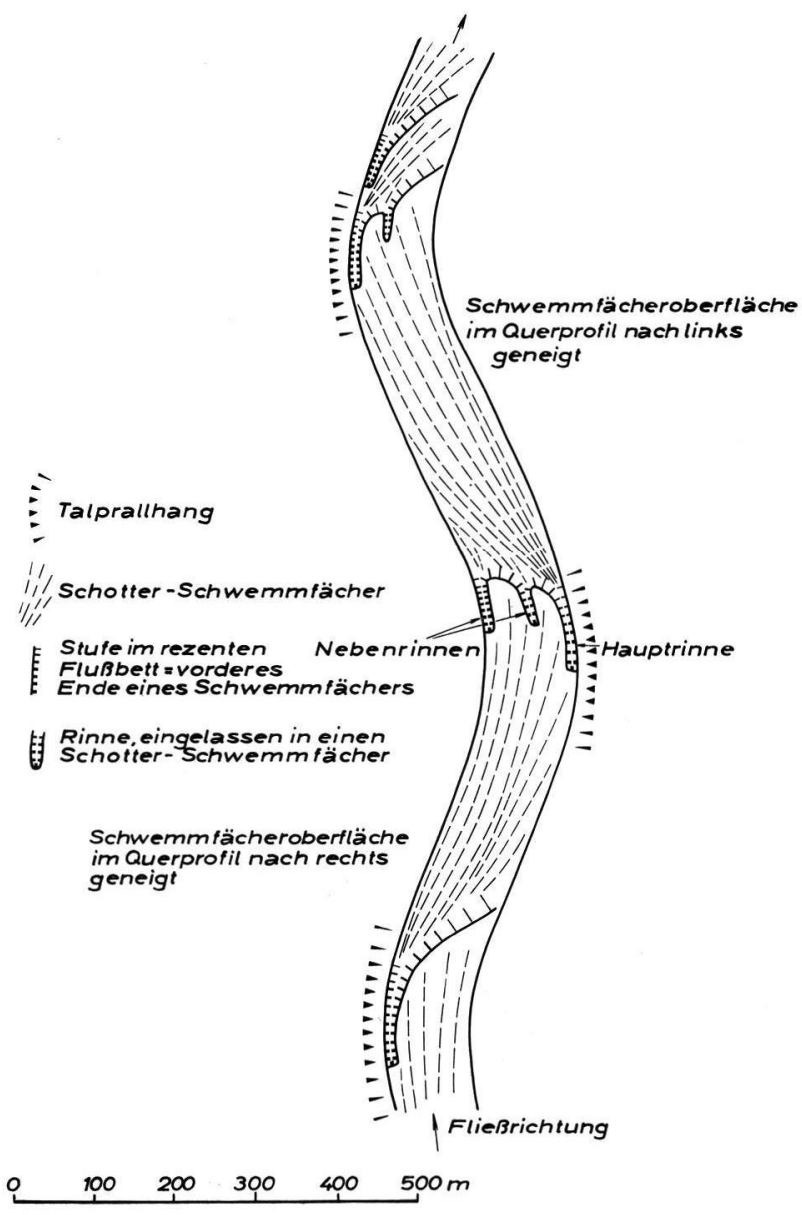

Abb. 10. Schotter-Schwemmfächer im Mittellauf des Oued Zigzaou. 
Die Beobachtungen in diesem Talabschnitt ergaben, daß sich die meist groben Schotter des Oueds, die in erster Linie durch Aufarbeitung der Schotter der "Jüngeren Akkumulation“, der "Hauptakkumulation“ und der Kalkkrusten dem Oued zur Verfügung stehen, in Form von mehrere $100 \mathrm{~m}$ langen und $20-80 \mathrm{~m}$ breiten Schotter-Schwemmfächern talab transportiert werden. Das vordere Ende eines Schotter-Schwemmfächers liegt dabei immer am Beginn einer Talkrümmung, wenn man die Schwemmfächer talab verfolgt. Die Oberfläche eines Schwemmfächers, der an seinem vorderen Ende 1-2-3 m Höhe erreichen kann, fällt regelmäßig sanft in Richtung auf den Prallhang der Talkrümmung ab (Abb. 10). In das vordere Ende jedes Schotter-Schwemmfächers greift in talaufwärtiger Richtung eine $0,5-1,5 \mathrm{~m}$ tiefe Hauptrinne am Prallhang des Oueds mehrere Dekameter weit in den nächst höher liegenden Schwemmfächer zurück. Nebenrinnen, die den Charakter von schmalen Erosionskanälen annehmen, können auch an der gegenüberliegenden Talseite oder in der Mitte des Schotter-Schwemmfächers in diesen rückschreitend zurückgreifen. Die Rinnen laufen auf der Oberfläche des nächsten, tiefer gelegenen Schwemmfächers aus. Infolge des Abfallens der Schwemmfächer-Oberfläche in Richtung auf den Prallhang und die hier entwikkelte Hauptrinne liegt bei einem Abkommen die größte Wassertiefe und höchste Fließgeschwindigkeit in diesem Bereich.

Der Schottertransport im rezenten Flußbett nimmt vom unteren Mittellauf (südlich bzw. südwestlich der Straße Mareth-Medenine) bis zum oberen Unterlauf ständig ab. Im unteren Unterlauf, also den untersten $7-8 \mathrm{~km}$ der Oued-Laufstrecke, ist ein Schottertransport völlig zum Erliegen gekommen. Hier kann nur noch sandiges und schluffiges Material transportiert werden.

In unmittelbarer Küstennähe wechselt in kurzen Zeitabständen die Ablagerung von blaugrauen, $7-15 \mathrm{~cm}$ dicken Schlicklagen mit ca. $5 \mathrm{~cm}$ mächtigen gelben Flugsanden ab.

Diese Beobachtungen geben den Hinweis, daß die Oueds im gleichen Talabschnitt, in dem noch zur Zeit der Bildung der „Hauptakkumulation“ grobe Schotter transportiert und abgelagert wurden, heute nicht mehr genügend Transportkraft für eine Schotterbewegung besitzen. Daraus dürfte auf eine heute hier existierende größere Aridität gegenüber der Zeit der „Hauptakkumulation“ zu schließen sein. Heute soll es nach Balland (1973) jährlich ca. 2 größere Abkommen der Oueds im Becken von Beni Zeltene und Toujane geben.

\section{Versuch einer klimatischen Deutung der Befunde}

Die in den Kapiteln 3-7 dargestellten und in Tab. 1 zusammengefaßten Befunde zum morphologischen Geschehen in der nördlichen Djeffara lassen u.E. nur wenige Schlüsse auf bestimmte klimatische Verhältnisse zu.

Die ältere (mittel- bis altpleistozäne und vorpleistozäne) Reliefgeschichte läßt sich anhand des überlieferten Formenschatzes kaum deutlich fassen. Es läßt sich lediglich aus den Kalkverbackungen von Schottern im Flußbett und auf niedrigen Felssockeln über dem Flußbett vermuten, daß bereits vor der „Hauptakkumulation“ Klimaverhältnisse vorgelegen haben müssen, die zu einer Kalkverbackung von Schottern und zu Kalkkrusten geführt haben und feuchter als die gegenwärtigen gewesen sein müssen. Denn heute konnten auf kalkhaltigen lockeren Schottern, die vor allem die untere Partie der "Jüngeren Akkumulation" aufbauen, keine Kalkkrustenbildungen oder Kalkverbackungen von Schottern festgestellt werden. Gegenwärtig läßt sich auch - etwa im Gegensatz zum südlichen Vorland des Djebel Chambi (vgl. Molle \& Brosche 1976) - am Top lößartiger Feinsedimente in der nördlichen Djeffara keine Kalkkrustenbildung feststellen.

Die Kalkverbackung von Schottern der „Hauptakkumulation“ kann nach oder während der Ablagerung der „Hauptakkumulation“ erfolgt sein. Sicher ist, daß nach Ablagerung der "Hauptakkumulation", bei deren Bildung die mechanische Gesteinsa $\mathrm{ufbereitung}$ ein bedeutendes und seitdem nicht mehr erreichtes Ausmaß angenommen hat, Klimaverhältnisse geherrscht haben müssen, die eine Kalkverbackung von Schottern und Kiesen, aber auch eine Kalkkrustenbildung am Top der Schotter ermöglichten (z. T. Bildung lamellenartiger Krusten oder dicker, fast reiner Kalkkrusten). Hierbei dürf- 
ten höhere Niederschläge als sie gegenwärtig in der nördlichen Djeffara anzutreffen sind, gefallen sein. Sie dürften wenigstens $350-400 \mathrm{~mm}$ erreicht haben - gegenüber $100-200$ mm Niederschlag heute. Bezüglich des Mechanismus der Kalkverbackung und Kalkkrustenbildung vermuten wir, daß nicht nur nach dem Aufbau der Hauptakkumulation eine Kalkverbackung von Schottern und Kalkkrustenbildung im Top der „Hauptakkumulation“ möglich war, sondern daß auch während der folgenden Zerschneidung der „Hauptakkumulation “ im Schotterkörper kalkgesättigte Wässer auf das jeweiligeNiedrigwasserbett der Hauptoueds eingestellt waren. Diese ermöglichten die Verbackung weiter Teile der "Hauptakkumulation“. Die Frage, ob auch bereits wä h $\mathrm{r}$ e $\mathrm{n}$ d der akkumulativen Aufhöhung der "Hauptakkumulation“ eine Verbackung durch kalkhaltige Wässer stattfand, die in den Schottern zirkulierten, vermögen wir nicht zu beantworten.

Eine gewisse paläoklimatische Ausdeutung erlaubt auch die Tatsache, daß in der Zeit der "Hauptakkumulation“ (und vielleicht schon vorher in der Zeit einer älteren Akkumulation, die sich an der Küste als 15-16-m-Niveau mit kalkverkrusteten Schottern darstellt) grobe Schotter wenigstens $7-8 \mathrm{~km}$ weiter in das Matmata-Vorland transportiert worden sind als heute Schotter im rezenten Flußbett bewegt werden können. Dabei haben sich die Gefällsverhältnisse im Unterlauf des Oued Zigzaou nicht geändert. Sie betrugen und betragen etwa $0,3 \%$ in den untersten $12 \mathrm{~km}$ Laufstrecke. Der Differenzbetrag von 7-8 km dürfte ein Mindestbetrag sein, denn es ist nicht bekannt, ob und wie weit die "Hauptakkumulation" ehemals weiter nach NE gereicht hat. Die Schotter der "Hauptakkumulation“ erreichten dabei in der Nähe der heutigen Küste mindestens noch $4 \mathrm{~m}$ Mächtigkeit und dürften schon aufgrund dieser Tatsache ehemals weiter nach NE transportiert worden sein. Sofern die das 15-16-m-Niveau an der Küste aufbauenden Schotter zu der "Hauptakkumulation“ gehören - was nicht geklärt werden konnte - hätte diese sogar in der Nähe der heutigen Küste eine Mächtigkeit von ca. $10 \mathrm{~m}$ erreicht. Setzt man einmal voraus, daß zur Zeit der „Hauptakkumulation“ die starken Niederschläge und Abkommen der Flüsse wie heute episodisch bzw. periodisch erfolgt sind, so scheint der Schluß berechtigt zu sein, daß die Abkommen zur Zeit der „Hauptakkumulation" - und zur Zeit der Ablagerung der möglicherweise von ihr zu unterscheidenden Schotterlagen im 15-16-m-Niveau - intensiver als heute gewesen sein müssen. Um diese Vermutung abzusichern, wäre es allerdings erforderlich, die nördlichsten Vorkommen von Schottern und Kiesen in den Oued-Betten systematisch aufzunehmen und sie mit dem Zustand nach 10 oder 20 Jahren zu vergleichen.

Die Anwehung und Ablagerung der Schluffe von Matmata wird von Balland (1973) in eine gegenüber heute feuchtere Zeit gestellt, weil nach ihrer Ansicht erst dann eine entsprechende Vegetationsdecke bestanden haben kann, die den Schluff auffangen konnte.

Nach unseren Befunden ist das ganze Geschehen der Schluffbildung und Überprägung differenzierter zu betrachten. Zunächst ist gegenwärtig zumindest im Sommer ein Sandund Staubtransport im ganzen Untersuchungsgebiet festzustellen. Die Verbreitungsgebiete der größten Schluff-Mächtigkeiten (Bergland von Matmata und Gebirgsrand) sind heute anthropogen stark überformt, d.h. sie werden über längere Zeit des Jahres ackerbaulich genutzt und damit ihres natürlichen Pflanzenkleides beraubt. Es ist u. E. durchaus denkbar, daß bei einem natürlichen Pflanzenkleid (Wüstensteppe, Steppe) auch heute noch trotz recht geringer Niederschläge - die aber im Bergland von Matmata etwas gegenüber der Djeffara-Ebene ansteigen dürften (Matmata bei $400 \mathrm{~m}$ ü. M. erhält etwa $240 \mathrm{~mm}$ durchschnittlichen Jahresniederschlag) - eine Schluff- und Flugsandakkumulation am Gebirgsrand und im Gebirge stattfinden kann.

Mit einiger Sicherheit läßt sich allerdings die fossile „Kalkbraunerde“, die den oberen gelblich-rötlichen Schluff gliedert, dahingehend klimatisch ausdeuten, daß zu ihrer Bildung ein deutlich gegenüber heute feuchteres Klima notwendig war; denn heute findet keine 
Bodenbildung im Top der Schluffe statt. Diese Interpretation wird auch dadurch unterstützt, daß an wenig erniedrigten Schluffwänden im oberen gelblich-rötlichen Teil keine Kalkknollen, Kalkkindeln oder Kalklamellen auftreten, wohl aber unterhalb der fossilen "Kalkbraunerde“. Das spricht gleichfalls für ehemals höhere Feuchtigkeit und eine ehemalige stärkere Wasserzirkulation im Schluff während der Bodenbildungsphase, in der Kalk in höheren Schlufflagen gelöst und profilabwärts wieder abgelagert wurde. Der Zeitraum der Bildung der fossilen „Kalkbraunerde“ dürfte also mit einiger Sicherheit mit einer morphologischen Stabilitätszeit i. S. von Rohdenburg \& SAbelberg (1969a, 1969b, 1973) zusammenfallen. Die fossile "Kalkbraunerde“ konnte sich damals selbst noch auf 10 bis $15^{\circ}$ geneigten Hängen halten.

Vergleicht man das Material der „Jüngeren Akkumulation“ mit dem Material, das die „Hauptakkumulation“ aufbaut, so dürfte der Schluß zulässig sein, daß die Abkommen und die Transportkräfte während der Zeit der „Hauptakkumulation “ wesentlich intensiver gewesen sein müssen als während der Zeit der „Jüngeren Akkumulation“. Während der Ablagerung der gröberen Basissedimente der „Jüngeren Akkumulation “ (Sande, Kiese, Schotter) waren dagegen die Abkommen noch etwas kräftiger als in der Zeit danach. Das Milieu während der Sedimentation der feinen Ablagerungen der "Jüngeren Akkumulation" dürfte sich kaum mit einem starken Abfluß vereinbaren lassen, sondern eher mit einem ruhigen Abflußvorgang. Ob bei der Ablagerung dieser Sedimente ein periodisch-episodischer oder ein über längere Zeit gleichmäßig anhaltender Wasserabfluß herrschte, läßt sich nicht beurteilen.

Mit Sicherheit kann dagegen geschlossen werden, daß während der Ablagerung des Großteils der "Jüngeren Akkumulation“ reichlich feines Material angeliefert wurde. Die Sedimente können entweder vorwiegend durch äolischen Transport in die nördliche Djeffara gelangt und danach in die Haupt-Oueds fluviatil weiter transportiert worden sein oder sie lassen sich aus den Schluffen von Matmata herleiten (Tab. 1). Dabei muß jedoch die Frage offenbleiben, ob die Feinsedimente der "Jüngeren Akkumulation “ bereits während der Ablagerung des gesamten Schluffpaketes sedimentiert wurden oder ob sie erst nach der Ablagerung der Schluffe von Matmata als Abtragungsprodukte dieser Schluffe entstanden sind. Während der Sedimentation des mächtigen Schluffpakets im Bergland von Matmata hat, wie die Aufschlüsse zeigen, keine linienhafte Erosion in diesen Schluffen stattgefunden.

Die Schluffe im Top der "Jüngeren Akkumulation“ des mittleren und unteren Mittellaufes sowie des Unterlaufes der Oueds deuten auf eine Fortdauer der Schluffbildung in Matmata-Vorland nach der Bildung der "Jüngeren Akkumulation“ hin.

\section{Die zeitliche Einordnung der Formbildungsphasen}

Eine detaillierte Darstellung der zeitlichen Einordnung der Formbildungsphasen im Untersuchungsgebiet geht aus Tabelle 1 hervor. Es lassen sich eindeutig rezente und subrezente Akkumulationen und Einschneidungsphasen von dem Formenschatz der gut datierten "Jüngeren Akkumulation" trennen, deren Aufbau im Zeitraum zwischen 8600 B.P. und 7700 B.P. als gesichert angesehen werden kann. Da unterhalb und oberhalb der ${ }^{14} \mathrm{C}$ datierten Holzkohlenbänder noch $1,0 \mathrm{bzw}$. 1,6-1,7 m mächtige Sedimente auftreten (Abb.6), dürfte der Aufbau der "Jüngeren Akkumulation" noch einen etwas längeren Zeitraum als den oben angegebenen in Anspruch genommen haben. Die „Jüngere Akkumulation" weist stellenweise ein um mehrere Meter erniedrigtes $Z_{\text {wischenniveau auf, an }}$ dessen Oberfläche neolithische Artefakte gefunden wurden. Dadurch ist erwiesen, daß die flächenhafte Überformung der "Jüngeren Akkumulation“ kurz nach Abschluß der Akkumulation erfolgt sein muß. 
Wie bereits im vorigen Kapitel dargelegt, ließ sich bisher das Alter der Schluffe im Bergland von Matmata und am Gebirgsrand nicht durch ${ }^{14} \mathrm{C}-\mathrm{Daten}$ belegen. Allerdings liegen zwei ${ }^{14} \mathrm{C}-$ Daten aus Deck-Schluffen im Vorland des Matmata-Berglandes vor (Hv 5401: $8975 \pm 215$ B.P. und Hv 5404: $8315 \pm 120$ B.P., Abb. 6), die an Schneckengehäusen gewonnen wurden. Bis jetzt konnte noch nicht geklärt werden, in welcher zeitlichen Beziehung die Deck-Schluffe des Vorlandes zu den mächtigen Schluffen im Bergland stehen. Hervorzuheben ist, daß im Hauptverbreitungsgebiet der Schluffe von Matmata (Gebirge und Gebirgsrand) die "Jüngere Akkumulation“ als Flußterrasse fehlt. Aus den im vorigen Kapitel angegebenen Überlegungen dürfte zumindest ein Teil der Schluffe von Matmata älter als die "Jüngere Akkumulation“ sein, zumal die fossile Kalkbraunerde im Schluff von Matmata auf einen längeren, durch eine feuchtere Phase unterbrochenen Ablagerungsprozeß hindeutet. Ein rißzeitliches Alter der Schluffe von Matmata, wie es BALland (1973) annimmt, erscheint uns jedoch zu hoch. Wahrscheinlicher ist dagegen ein würmzeitliches bis frühholozänes Alter der Schluffe von Matmata, wobei die fossile Kalkbraunerde möglicherweise in einen spätglazialen feuchteren Abschnitt des Würm zu stellen ist. Dies ist ein Zeitraum, der nach mehreren vergleichenden Untersuchungen von BRUNNACKer \& LožEk (1969), Brunnacker, Basler, LožEk, Beug \& Altemüller (1969), BrunNACKer, Altemüller \& Beug (1969), Brunnacker (1973) und Brunnacker (1974) wahrscheinlich durch größere Feuchtigkeit und z. T. durch die Bildung von Paläoböden in Tunesien gekennzeichnet war.

Diese zeitliche Einstufung steht auch im Einklang mit dem ${ }^{14} \mathrm{C}$-Datum einer fast reinen Kalkkruste am Top der „Hauptakkumulation“ (Hv 5402, $21385 \pm 235$ B.P.) des Oued Zigzaou. Aus diesem Datum dürfte bei allem Vorbehalt hervorgehen, daß die darunter liegende "Hauptakkumulation" zumindest würmzeitlich, vielleicht sogar älter ist. Ein mindelzeitliches Alter der „Hauptakkumulation“, wie es BALLAND (1973) annimmt, erscheint uns ebenfalls zu hoch.

Eine zeitliche Einstufung des Schotterniveaus, das 7-10 m über dem Top der „Hauptakkumulation" liegt, ist uns ebensowenig möglich wie die zeitliche Einordnung des 40 bis $50 \mathrm{~m}$ ü. M. gelegenen Küstenniveaus in Pont-Mergeln.

\section{Schriftenverzeichnis}

Balland, V.: Morphogenèse Quaternaire dans les Monts de Matmata. - Maghreb et Sahara, Etudes Géographiques offerts à Jean Despois. - Société de Géographie, Acta Geographica, Paris 1973.

Birot, P.: La Géomorphologie Quaternaire dans les régions méditerranéennes (principalement d'après les publications françaises). - Etudes françaises sur le Quaternaire, presentées à l'occasion du VIIIe Congrès International de l'INQUA, 173-192, Paris 1969.

Brosche, K.-U. \& Molle, H.-G.: Geomorphologische und klimageschichtliche Studien in Süd- und Zentraltunesien. - Z. Geomorph., N. F., Suppl. Bd. 20, Berlin - Stuttgart 1976 (im Druck).

Brosche, K.-U., Molle, H.-G. \& Schulz, G.: Geomorphologische Untersuchungen im östlichen Kroumirbergland (Nordtunesien, Gebiet östlich von Tabarka. - Unveröffentl. Manuskript, 20 Seiten, Berlin 1976.

Brunnacker, K.: Affleurements de Loeß dans les Régions nord-méditerranéennes. - Revue Géogr. physique et Géol. dynamique 9, 325-334, Paris 1969.

- : Einiges über Löß-Vorkommen in Tunesien. - Eiszeitalter u. Gegenwart 23/24, 89-99, Ohringen 1973.

- : Lösse und Paläoböden der letzten Kaltzeit im mediterranen Raum. - Eiszeitalter u. Gegenwart 25, 62-95, Ohringen 1974.

Brunnacker, K., Altemüller, H.-J. \& Beug, H.-J.: Das Profil von Kitros in Nord-Griechenland als Typusprofil einer mediterranen Lößprovinz. - Eiszeitalter u. Gegenwart 20, 90-110, Öhringen 1969. 
Tabelle 1. Abfolge der Reliefstadien und Formungsphasen im nordöstlichen Matmata-Vorland (Gebiet des Oued Zigzaou, Oued Ez Zeuss und Oued Oum Ez Zessar)

Alter 6 )

Unterlauf

$40-55 \mathrm{~m}$ ü.N.N. Niveau im Pont (ohne Schotter)

Erosion 1)

\section{$15-16 \mathrm{~m}$ ü.N.N. Niveau}

mit einer maximal

3-4 m mächtigen Schot-

terdecke (Kalkverbackene

Schotter)

Erosion

Mittellauf

Oberlauf

$\begin{array}{ll}\text { Niveau mit Schotterdecke } & \text { NW, Niveau mit } \\ & \begin{array}{l}\text { Blöcken und } \\ \text { Grobschutt }\end{array} \\ & \text { (Kalkverbackung) }\end{array}$

Hv 5402: $21385 \pm 235$

(Kalkkruste der „Haupt-

akkumulation" b.Mareth;

Hv 5403: $4310 \pm 170$ :

Datierung der marinen

Schnecke Murex trun-

culus auf Oberfläche des

$7-8 \mathrm{~m}$ Niveaus

Kalkverbackene

7-8 m über NN Niveau

über Schottern der

"Hauptakkumulation"

Kalkverbackene

Schotter)

Erosion

(in Haupttälern)

\begin{abstract}
$9-10 \mathrm{~m}$ über NW
Niveau der „Haupt-

akkumulation" (Kalk-

verbackene Schotter)
\end{abstract}

Errossion

Errosion

Vorkommen von Schneckengehäusen 5 )

$\begin{array}{lll}\text { Erosion } & \text { Erosion (in Haupt- } & \text { Eros ion ? } \\ \text { (in Haupttälern) } & \text { tälern, Anlage breiter } & \text { Muldentäler auf der } \\ & \text { Oberfäche der „Haupt- } & \text { akkumulation") }\end{array}$

Bergland von Matmata und
Hv 5566: $8600 \pm 150$

(Holzkohle) Mittellauf Hv 5400: $7775 \pm 340$ (Holzkohle) Mittellauf

Hv 5401: $8975 \pm 215$ (Schnecken) 4) Mittellauf $\mathrm{Hv} 5404: 8315+120$ (Schnecken) 4) Unterlauf
5-10 m mächtige „Jüngere Akkumulation

(Sand, Kies, mit Holz-

kohlen- u. Lehmbändern)

-ー-ー- kohlen-u. Lehmbändern)

Decke aus Schluffen

\section{Erosion}

Zwischenniveau in $3,5 \mathrm{~m}$ über NW in Küstennähe ohlen-u. Lehmbändern)
Decke aus Schluffen Erosion

4-6 m mächtige "Jünere Akkumulation (Sand, Kies, mit Holz Erosion

Zwischenniveau mit neolithischen Artefakten an der Oberfläche unmittelbarer Randbereich:

$4-5 \mathrm{~m}$ über $\quad 8-10 \mathrm{~m}$ über

NW, Niveau NW, Niveau der

der „Haupt- "Hauptakkumu-

akkumulation" lation" (Kalk-

(Kalkverbak- verbackene

(Kalkverbak- verbacken

Untere graugelbliche

Schluffe mit Schuttstückchen

Bodenbildung (Lamellen-

? $\uparrow$ Kalkkruste unter „Kalkbraunerde“, Kalkkindel-

bildung unter „Kalkbraunerde")

? obere, ockerfarbene Schluffe (z. T. mit etwas Schutt)

Erosion marine Schnecke Murex trunculus auf dem $15-16 \mathrm{~m}$

trunculus auf dem 7-8 $\mathrm{m}$ Niveau in Küstennähe Niveau in Küstennähe

Schneckengehäuse in der Schluffdecke der „Jüngeren Akkumulation":

Probe 99 (Mittellauf) (Alter: $8975 \pm 215$ ):

179 Exemplare von Sphincterocbila candidissima

(Draparnaud)

(west-mediterran) 
Erosion bis unter NW Erosion bis unter NW Erosion ungefähr bis zum heutigen bzw. etwas über dem heutigen NW

subrezent

rezent
Sand- u. Fein- Kies, materialtransport Schot-

im Flußbett, ter

Ablagerung von

Schlick und Flugsand

im Wechsel in Küsten-

nähe, Deflation

Kupsten, Dünen.
"Jüngste Akkumulation “

bis $1-2 \mathrm{~m}$ über NW

(Ursache: Brückenbau)

$$
\text { Erosion }
$$

Schwemmfächer grobe

ausgroben Schot-

Schottern mit

Schot-
ter

Rinnensystem

Kupsten im Hoch-

wasserbett,

Sandverwehung
"Jüngste Akkumulation": Fein-

materialablagerungen oberhalb

künstlich angelegter Steinwälle

(geht heute zum Teil weiter)

Blöcke und grobe Schotter auf An-

stehendem im Flußbett. Kerben

im Schluff, Feinmaterialverschwem-

mung, Schuttaufbereitung, Schutt-

verlagerung auf Steilhängen, Ein-

wehung von Schluff aus Grand Erg

Orientale während des Chehili. 3)
Probe 125 (Unterlauf)

(Alter: $8315 \pm 120$ ):

267 Exemplare von Sphinc-

terochila candidissima

(Draparnaud)

1 Exemplar von Eobania

vermiculata (O. F. Müller)

(zirkummediterran)

Kerbbildung im Rand- Kerbtälchen bereich des Haupttales

1) Diese Erosionsphase ging bis zur Basis der Schotterdecke des 15-16 m Niveaus bzw. bis zur Basis der „Hauptakkumulation“, je nachdem ob es sich um zwei oder lediglich um eine Akkumulationsphase bei diesen Schotterablagerungen handelte; in der Tabelle wurde der 1. Fall dargestellt, vgl. dazu aber den Text.

2) $\mathrm{NW}=$ Niedrigwasserbett.

3) Chehili $=$ Chili $=$ Samum in Marokko und Algerien.

4) Korrektur notwendig, es sind um 1000 Jahre zu hohe Alter möglich (vgl. Text).

5) Die Schneckengehäuse wurden bestimmt von Dr. H. Schütt, Düsseldorf-Benrath.

6) Die ${ }^{14} \mathrm{C}$-Daten wurden im ${ }^{14} \mathrm{C}$ - und $3 \mathrm{H}$-Labor des Niedersächsischen Landesamtes für Bodenforschung ermittelt (Leiter: Dr. M. A. Geyh). 
Brunnacker, K., Basler, Dj., Ložek, V., Beug, H.-J. \& Altemüller, H.-J.: Zur Kenntnis der Lösse im Neretva-Tal. - Jb. Geol. Paläont., Abh. 132, 127-154, Stuttgart 1969.

Brunnacker, K. \& Ložek, V.: Löß-Vorkommen in Südost-Spanien. - Z. Geomorph., N. F. 13, 297-316, Berlin-Stuttgart 1969.

Coque, R.: La Tunisie Présaharienne. Etude géomorphologique, Paris 1962.

Houérou le, H. N.: Contribution à l'étude des sols du Sud Tunisien. - Ann. Agron., Série A, 3, 241-308, Paris 1960.

Mensching, H.: Bericht und Gedanken zur Tagung der Kommission für Periglazialforschung in der I.G.U. in Marokko am 19.-31. Okt. 1959. - Z. Geomorph., N. F. 4, 159-170, BerlinStuttgart 1960.

— : Morphologie des südtunesischen Schichtstufenlandes. - Bull. Soc. hellén. Géogr., 152-161, Athen 1963.

— : Zur Geomorphologie Südtunesiens. - Z. Geomorph., N.F. 8, 424-439, Berlin 1964.

Molle, H.-G. \& Brosche, K.-U.: Morphologische und klimageschichtliche Untersuchungen im südöstlichen Vorland des Djebel Chambi und des Djebel Mrhila in Zentraltunesien. - Die Erde 107, Berlin 1976 (im Druck).

Rathjens, C.: Löß in Tripolitanien. - Z. Ges. Erdkde. 1928, 211-228, Berlin 1928.

Rohdenburg, H.: Morphodynamische Aktivitäts- und Stabilitätszeiten statt Pluvial- und Interpluvialzeiten. - Eiszeitalter u. Gegenwar 21, 81-96, Öhringen 1970.

- : Einführung in die klimagenetische Geomorphologie - anhand eines Systems von Modellvorstellungen am Beispiel des fluvialen Abtragungsreliefs, 350 S., Gießen 1971.

Rohdenburg, H. \& Meyer, B.: Zur Feinstratigraphie und Paläopedologie des Jungpleistozäns nach Untersuchungen an südniedersächsischen und nordhessischen Lößprofilen. - Mitt. d. Dtsch. Bodenkundl. Ges. 5, 1-137 (= Göttinger Bodenkundl. Ber. 2), Göttingen 1966.

Rohdenburg, H. \& SAbelsberg, U.: „Kalkkrusten“ und ihr klimatischer Aussagewert - neue Beobachtungen aus Spanien und Nordafrika. - Göttinger Bodenkundl. Ber. 7, 3-26, Göttingen 1969 (1969 a).

- : Zur landschaftsökologisch-bodengeographischen und klimagenetisch-geomorphologischen Stellung des westlichen Mediterrangebietes. - Göttinger Bodenkundl. Ber. 7, 27-47, Göttingen 1969 (1969 b).

Rohdenburg, H. \& Sabelberg, U.: Quartäre Klimazyklen im westlichen Mediterrangebiet und ihre Auswirkungen auf die Relief- und Bodenentwicklung. - Catena, 1, 71-180, Gießen 1973.

Schwegler, E.: Bemerkungen zum Vorkommen von Löß im libyschen und tunesischen Gebiet. N. Jb. Min. etc., Mh. Abt. B, 1944, 10-17, Stuttgart 1944.

Manuskript eingeg. 6. 6. 1975.

Anschrift der Verf.: Dr. K.-U. Brosche u. Dr. H.-G. Molle, Geomorpholog. Labor der FU Berlin, Altensteinstraße 19, 1000 Berlin 33. 